K. Ueno

Nagoya Math. J.

Vol. 108 (1987), 15-51

\title{
ON PSEUDOELLIPTIC SYSTEMS OF FIRST ORDER DIFFERENTIAL EQUATIONS
}

\author{
KAZUSHIGE UENO
}

\section{Contents}

§1. Defining equations of automorphism pseudogroups

§2. Differential invariants of differential operators of infinite type

$\S 3$. Invariant structures of differential operators of infinite type

$\S 4$. Geometric structures of differential operators of infinite type

§5. Differential equations whose invariant structures are given by famillies of pseudoelliptic systems

§6. Foliations and different:al equations

\section{Introduction}

In the study of elliptic differential operators of second order, we found that the automorphism pseudogroups are of finite type ([4]). However this fact takes a complete change in elliptic differential operators of first order.

So as to make the objects which can be dealt with clear, we introduce the concept of pseudoellipticity of first order differential operators (Definition 1.1), which is naturally satisfied by first order elliptic differential operators.

Here we restrict our considerations to differential operators defined on the jet space $J^{1}\left(R^{2}, R^{2}\right)$. We can classify first order pseudoelliptic differential operators into two types: One is of finite type and the other is of infinite type.

If a differential operator $P(x, D)$ is of finite type, the automorphism pseudogroup is generated by the maximal subgroup of the affine transformation group whose linear part is just the center of $G L(2, R)$ (Corollary 4.2). Thus in this case by similar arguments to elliptic differential operators of second order, the differential operator $P(x, D)$ admits a

Received July 19, 1985. 
transversally projective structure on the space of integral points $I(P)$ of $P(x, D)$ (cf. [4]).

If a differential operator $P(x, D)$ is of infinite type, the system of defining equations of the automorphism pseudogroup $\mathfrak{U}(P)$ is of CauchyKowalewski type (Theorem 4.1, Definition 6.1).

In this paper we develop an invariant theory of first order pseudoelliptic differential operators of infinite type. In particular, we determine the canonical invariant generator of a first order pseudoelliptic system of differential equations of infinite type (Theorem 9.1). Consequently we see that, under the differentiability of class $C^{\omega}$, a first order differential operator $P(x, D)$ is pseudoelliptic and of infinite type with the structure vector $(a, b)$ if and only if it is $\mathfrak{A}(P)$-automorphic and $\mathfrak{A}(P)=\mathfrak{P}_{(a, b)}$ where $\mathfrak{P}_{(a, b)}$ is a pseudogroup determined by the vector $(a, b)$ (Theorem 12.1).

From this standpoint we study a generalization of pseudoelliptic differential operators of infinite type and prove that, if a single differential equation $P$ is "decomposed" into a family of pseudoelliptic systems of differential equations of infinite type with the structure vector $(a, b)$ by a rational expression with two variables, then the automorphism pseudogroup $\mathfrak{U}(P)$ coincides with that of each pseudoelliptic system of differential equations (Theorem 16.2). These theorems (Theorem 12.1 and 16.2) are very interesting from the viewpoint of geometric foliations. We state these theorems in terms of foliations (Proposition 17.2, 18.1) and attempt to connect our study of differential equations with that of transversal structures of foliations.

In Section 1, the system of defining equations of the automorphism pseudogroup $\mathfrak{X}(P)$ of a first order pseudoelliptic differential operator $P(x, D)$ is determined (Theorem 4.1).

In Section 2, we calculate a fundamental system of differential invariants of $\mathfrak{U}(P)$ for a pseudoelliptic differential operator $P(x, D)$ of infinite type (Proposition 8.1).

In Section 3, we find a canonical generator of a pseudoelliptic system of differential equations of infinite type $P(x, D) u=o$ (Theorem 9.1).

In Section 4 , we state a characterization of a pseudoelliptic differential operator $P(x, D)$ of infinite type by the standpoint of the automorphism pseudogroup $\mathfrak{U}(P)$ (Theorem 12.1).

In Section 5, we study a generalization of a pseudoelliptic system of differential equations of infinite type from an invariant point of view 
(Theorem 16.2).

In Section 6, we study a structure of a pseudoelliptic system of differential equations of infinite type or of its generalization from the viewpoint of foliation (Proposition 17.2, 18.1).

A generalization of our study in the sapce $J^{1}\left(R^{2}, R^{2}\right)$ to that in the space $J^{1}\left(R^{n}, R^{m}\right)$ and a general study of foliations associated with first order pseudoelliptic differential operators or their families remain to be done as interesting problems.

\section{§1. Defining equations of automorphism pseudogroups}

1. Let $E$ be a vector space over $R$ and denote by $\Gamma(n, E)$ the set of local $C^{\infty}$-maps of $R^{n}$ to $E$. Let $F$ be another vector space over $R$ and denote by $\operatorname{Hom}(E, F)$ the vector space over $R$ of linear maps of $E$ to $F$. Then for the canonical coordinate system $\left\{x_{1}, \cdots, x_{n}\right\}$ on $R^{n}$, a differential operator of $\Gamma(n, E)$ to $\Gamma(n, F)$ of order 1 is an expression

$$
P(x, D)=\sum_{k=1}^{n} a_{k}(x) D_{k}+b(x)
$$

where $D_{k}=\partial / \partial x_{k}$ and $a_{k}(x)$ and $b(x)$ are $\operatorname{Hom}(E, F)$-valued $C^{\infty}$-functions on $R^{n}$.

In this paper we deal with only these differential operators of order 1 .

To this differential operator $P(x, D)$ there corresponds the differential operator

$$
P_{\langle 1\rangle}(x, D)=\sum_{k=1}^{n} a_{k}(x) D_{k}
$$

called the principal part of $P(x, D)$.

Definition 1.1. A differential operator $P(x, D)=\sum_{k=1}^{n} a_{k}(x) D_{k}+b(x)$ of $\Gamma(n, E)$ to $\Gamma(n, F)$ is said to be pseudoelliptic at $x \in R^{n}$ if $\operatorname{dim} E=\operatorname{dim} F$ and $\operatorname{det} a_{k}(x) \neq 0$ for $k=1, \cdots, n$.

Denote by $J^{1}(n, k)$ the 1-jet space of local maps of $R^{n}$ to $R^{k}$ and assume that $k=\operatorname{dim} E$. Then for any differential operator $P(x, D)$ it is easy to see that there exists a unique map $\sigma: J^{1}(n, k) \rightarrow F$ such that $P(x, D) f=\sigma\left(j_{x}^{1}(f)\right)$ for any element $f \in \Gamma(n, E)$ where $j_{x}^{1}(f)$ is the 1 -jet of $f$ with the source $x$.

Denote by $0 \in F$ the zero of $F$ and set $I(P)=\sigma^{-1}(0) \subset J^{1}(n, k)$. Let $\Im(P)$ be the set of elements $f \in \Gamma(n, E)$ satisfying $P(x, D) f=0$ and set 
$S(P)=\left\{j_{x}^{1}(f) ; x\right.$ belongs to the domain of $f$ and $\left.f \in \subseteq(P)\right\} \subset J^{1}(n, k)$. Then, in general, we have $S(P) \subset I(P)$. Each element of $\subseteq(P)$ is called a solution of $P(x, D)$ and each point of $I(P)$ is called an integral point of $P(x, D)$.

Definition 1.2. A differential operator $P(x, D)=\sum_{k=1}^{n} a_{k}(x) D_{k}+b(x)$ is said to be pseudoinvolutive at $p \in I(P)$ if $S(P)=I(P)$ on a neighbourhood of $p \in J^{1}(n, k)$.

Note that, under the analyticity, any differential operator of order 1 which is pseudoelliptic at $x$ is psedoinvolutive at any point $p \in I(P)$ whose source $\alpha^{1}(p)=x$.

2. In the following we assume that $n=\operatorname{dim} E=\operatorname{dim} F=2$ and that any differential operator $P(x, D)=\sum_{k=1}^{2} a_{k}(x) D_{k}+b(x)$ under consideration is pseudoelliptic at a point $x \in R^{2}$ and pseudoinvolutive at any point $p \in$ $I(P)$ with $\alpha^{1}(p)=x$.

Choosing a coordinate system $\left\{u_{1}, u_{2}\right\}$ (resp. $\left\{v_{1}, v_{2}\right\}$ ) on $E$ (resp. $F$ ), we can set $a_{k}(x)=\left(a_{k}^{i j}(x)\right)_{1 \leq i, j \leq 2}$ and $b(x)=\left(b^{i j}(x)\right)_{1 \leq i, j \leq 2}$. For any element $u \in \Gamma(2, E)$, if we set $P(x, D) u=v$, then we have

$$
v_{i}=\sum_{j=1}^{2} \sum_{k=1}^{2} a_{k}^{i j}(x)\left(\partial u_{j} / \partial x_{k}\right)+\sum_{j=1}^{2} b^{i j}(x) u_{j} \quad(i=1,2) .
$$

Let $\mathfrak{X}(P)$ denote the pseudogroup of all local transformations $\phi$ on $E$ such that, for any $u \in \widetilde{S}(P)$, if the composition $\phi \circ u$ is defined, then $\phi \circ u$ $\in \mathfrak{S}(P) . \quad \mathfrak{A}(P)$ is called the automorphism pseudogroup of $P(x, D)$. For such $\phi$ and $u$ we have

$$
\sum_{j=1}^{2} \sum_{k=1}^{2} a_{k}^{i j}(x)\left(\partial u_{j} / \partial x_{k}\right)+\sum_{j=1}^{2} b^{i j}(x) u_{j}=0 \quad(i=1,2)
$$

and

$$
\sum_{\ell=1}^{2} \sum_{j=1}^{2} \sum_{k=1}^{2} a_{k}^{i j}(x)\left(\partial \phi_{j}(u) / \partial u_{\ell}\right)\left(\partial u_{\ell} / \partial x_{k}\right)+\sum_{j=1}^{2} b^{i j}(x) \phi_{j}(u)=0 \quad(i=1,2) .
$$

Then by the pseudoellipticity of $P(x, D)$ at $x$ and by (2.1) and (2.2), we get

$$
\begin{aligned}
\operatorname{det} & \left(a_{1}(x)\right) \sum_{\ell=1}^{2} \sum_{j=1}^{2} a_{2}^{i j}(x)\left(\partial \phi_{j}(u) / \partial u_{\ell}\right)\left(\partial u_{\ell} / \partial x_{2}\right)+\sum_{j=1}^{2} a_{1}^{i j}(x)\left(\partial \phi_{j}(u) / \partial u_{1}\right) \\
& \times\left\{a_{1}^{12}(x)\left(\sum_{\ell=1}^{2} a_{2}^{2 \ell}(x)\left(\partial u_{\ell} / \partial x_{2}\right)+\sum_{\ell=1}^{2} b^{2 \ell}(x) u_{\ell}\right)\right. \\
- & \left.a_{1}^{22}(x)\left(\sum_{\ell=1}^{2} a_{2}^{1 \ell}(x)\left(\partial u_{\ell} / \partial x_{2}\right)+\sum_{\ell=1}^{2} b^{1 \ell}(x) u_{\ell}\right)\right\}
\end{aligned}
$$




$$
\begin{aligned}
& +\sum_{j=1}^{2} a_{1}^{i j}(x)\left(\partial \phi_{j}(u) / \partial u_{2}\right) \\
& \times\left\{a_{1}^{21}(x)\left(\sum_{\ell=1}^{2} a_{2}^{1 \ell}(x)\left(\partial u_{\ell} / \partial x_{2}\right)+\sum_{\ell=1}^{2} b^{1 \ell}(x) u_{\ell}\right)\right. \\
& \left.-a_{1}^{11}(x)\left(\sum_{\ell=1}^{2} a_{2}^{2 \ell}(x)\left(\partial u_{\ell} / \partial x_{2}\right)+\sum_{\ell=1}^{2} b^{2 \ell}(x) u_{\ell}\right)\right\} \\
& +\operatorname{det}\left(a_{1}(x)\right) \sum_{j=1}^{2} b^{i j}(x) \phi_{j}=0 \quad(i=1,2) .
\end{aligned}
$$

Since $P(x, D)$ is pseudoinvolutive at any point $p \in I(P)$ with $\alpha^{1}(p)=x$, the relation (2.3) holds identically on $I_{x, u}(P)=\left\{p \in I(P) ; \alpha^{1}(p)=x, \beta^{1}(p)=u\right\}$ where $\beta^{1}$ is the target projection of $J^{1}\left(R^{2}, E\right)$ onto $E . \quad I(P)$ is the set of points in $J^{1}\left(R^{2}, E\right)$ defined by $(2.1)$ and, on $I_{x, u}(P), \partial u_{1} / \partial x_{2}$ and $\partial u_{2} / \partial x_{2}$ are independent. Therefore from (2.3) we get

$$
\left\{\begin{array}{c}
\sum_{j=1}^{2} a_{2}^{i j}(x)\left(\partial \phi_{j} / \partial u_{\ell}\right) \operatorname{det}\left(a_{1}(x)\right) \\
\quad+\sum_{j=1}^{2} a_{1}^{i j}(x)\left(\partial \phi_{j} / \partial u_{1}\right)\left(a_{1}^{12}(x) a_{2}^{2 \ell}(x)-a_{1}^{22}(x) a_{2}^{1 \ell}(x)\right) \\
+\sum_{j=1}^{2} a_{1}^{i j}(u)\left(\partial \phi_{j} / \partial u_{2}\right)\left(a_{1}^{21}(x) a_{2}^{1 \ell}(x)-a_{1}^{11}(x) a_{2}^{2 \ell}(x)\right)=0 \\
(i, \ell=1,2), \\
\quad+\sum_{j, \ell=1}^{2} a_{1}^{h j}(x)\left(a_{1}^{21}(x) b^{1 \ell}(x)-a_{1}^{11}(x) b^{2 \ell}(x)\right) u_{\ell}\left(\partial \phi_{j} / \partial u_{2}\right) \\
+\operatorname{det}\left(a_{1}(x)\right) \sum_{j=1}^{2} b^{h j}(x) \phi_{j}=0, \quad(h=1,2) .
\end{array}\right.
$$

In the case $n=\operatorname{dim} E=\operatorname{dim} F=2$, this is the system of defining equations of $\mathfrak{A}(P)$ around $x \in R^{2}$ for a differential operator $P(x, D)$ which is pseudoelliptic at $x$ and pseudoinvolutive at any point $p \in I(P)$ with $\alpha^{1}(p)=x$.

The system of differential equations (2.4) seems to be "overdetermined". However later, in Theorem 4.1, we shall show that, if $P(x, D)=P_{\langle 1\rangle}(x, D)$ the system of defining equations $(2.4)$ of $\mathfrak{X}(P)$ comes to be "determined" under a "generic" condition. Throughout this paper we shall deal with pseudoelliptic operators $P(x, D)$ like that $P(x, D)=P_{\langle 1\rangle}(x, D)$.

3. Denote by $\mathscr{A}(E)$ the pseudogroup of all local transformations of $E$. The exceptional model which does not satisfy the "generic" condition 
(which is the condition $\operatorname{det} K^{i} \neq 0(i=1,2)$ in Theorem 4.1) is the case in the next proposition.

Proposition 3.1. Assume that a differential operator $P(x, D)$ is pseudoelliptic at $x_{0} \in R^{2}$ and pseudoinvolutive at any point $p \in I(P)$ with $\alpha^{1}(p)=x$ which is near to $x_{0}$. Furthermore assume that $P(x, D)=P_{\langle 1\rangle}(x, D)$ and $a_{2}^{11}\left(x_{0}\right) a_{2}^{12}\left(x_{0}\right) a_{2}^{21}\left(x_{0}\right) a_{2}^{22}\left(x_{0}\right) \neq 0$. Then $\mathfrak{U}(P)=\mathscr{A}(E)$ if and only if $a_{1}^{11}(x) / a_{2}^{11}(x)=$ $a_{1}^{12}(x) / a_{2}^{12}(x)=a_{1}^{21}(x) / a_{2}^{21}(x)=a_{1}^{22}(x) / a_{2}^{22}(x)$ around $x_{0}$. In this case for any $s \in$ $\mathfrak{S}(P)$, the rank of $s$ at each point of its domain near $x_{0}$ is smaller than 2 .

Proof. In the system of defining equations (2.4), the coefficients of $\partial \phi_{j} / \partial u_{1}$ are

$$
\begin{aligned}
& A_{j 1}^{i 1}(x)=a_{2}^{i j}(x)+\Lambda(x) a_{1}^{i j}(x)\left(a_{1}^{12}(x) a_{2}^{21}(x)-a_{1}^{22}(x) a_{2}^{11}(x)\right) \\
& \text { for } i=1,2 \text { and } \ell=1, \\
& A_{j 2}^{i 1}(x)=\Lambda(x) a_{1}^{i j}(x)\left(a_{1}^{12}(x) a_{2}^{22}(x)-a_{1}^{22}(x) a_{2}^{12}(x)\right) \\
& \text { for } i=1,2 \text { and } \ell=2
\end{aligned}
$$

and the coefficients of $\partial \phi_{j} / \partial u_{2}$ are

$$
\begin{aligned}
& A_{j 1}^{i 2}(x)=\Lambda(x) a_{1}^{i j}(x)\left(a_{1}^{21}(x) a_{2}^{11}(x)-a_{1}^{11}(x) a_{2}^{21}(x)\right) \\
& \text { for } i=1,2 \text { and } \ell=1, \\
& A_{j 2}^{i 2}(x)=a_{2}^{i j}(x)+\Lambda(x) a_{1}^{i j}(x)\left(a_{1}^{21}(x) a_{2}^{12}(x)-a_{1}^{11}(x) a_{2}^{22}(x)\right) \\
& \text { for } i=1,2 \text { and } \ell=2
\end{aligned}
$$

where $\Lambda(x)=\operatorname{det}\left(a_{1}(x)\right)^{-1}$. If we assume that $a_{1}^{11}(x) / a_{2}^{11}(x)=a_{1}^{12}(x) / a_{2}^{12}(x)=$ $a_{1}^{21}(x) / a_{2}^{21}(x)=a_{1}^{22}(x) / a_{2}^{22}(x)$, then we have $A_{j 1}^{i 1}(x)=A_{j 2}^{i 1}(x)=A_{j 1}^{i 2}(x)=A_{j 2}^{i 2}(x)=0$ and so the left hand sides of (2.4) are identically zero. This means that $\mathfrak{U}(P)=\mathscr{A}(E)$.

Conversely if $\mathfrak{U}(P)=\mathscr{A}(E)$, then $A_{j 1}^{i 1}(x)=A_{j 2}^{i 1}(x)=A_{j 1}^{i 2}(x)=A_{j 2}^{i 2}(x)=0$ from which we can easily obtain the equalities $a_{1}^{11}(x) / a_{2}^{11}(x)=a_{1}^{12} / a_{2}^{12}(x)=$ $a_{1}^{21}(x) / a_{2}^{21}(x)=a_{1}^{22}(x) / a_{2}^{22}(x)=\ell(x)^{-1}$.

Next we shall prove that the rank of any element $u \in \widetilde{S}(P)$ is smaller than 2 at each point of its domain $\delta(u)$. Any $u \in \mathbb{S}(P)$ satisfies

$$
\sum_{j=1}^{2} a_{1}^{i j}(x)\left(\partial u_{j} / \partial x_{1}\right)+\ell(x)\left(\sum_{j=1}^{2} a_{1}^{i j}(x)\left(\partial u_{j} / \partial x_{2}\right)\right)=0, \quad(i=1,2) .
$$

By the pseudoellipticity of $P(x, D)$, $\operatorname{det}\left(a_{1}(x)\right) \neq 0$. Therefore (3.1) implies that, at each point $x \in \delta(u)$, the vectors ${ }^{t}\left(\partial u_{1} / \partial x_{1}, \partial u_{2} / \partial x_{1}\right)$ and ${ }^{t}\left(\partial u_{1} / \partial x_{2}, \partial u_{2} / \partial x_{2}\right)$ are linearly dependent, that is, the rank of $u$ at $x \in \delta(u)$ is smaller than 2. This completes the proof of Proposition 3.1. 
4. Let us again consider a differential operator $P(x, D)=P_{\langle 1\rangle}(x, D)$ which is pseudoelliptic at $x_{0} \in R^{2}$ and pseudoinvolutive at any $p \in I(P)$ with $\alpha^{1}(p)=x$ which is near to $x_{0}$. We set

$$
\begin{array}{r}
K_{j \ell}^{i}(x)=(2-\ell) a_{2}^{i j}(x)+\Lambda(x) a_{1}^{i j}(x)\left(a_{1}^{12}(x) a_{2}^{2 \ell}(x)-a_{1}^{22}(x) a_{2}^{1 \ell}(x)\right), \\
(1 \leq i, j, \ell \leq 2) .
\end{array}
$$

Then, since $b^{i j}(x)=0$ for any $i, j$, the system of defining equations (2.4) around $x_{0}$ is written by the form

$$
\sum_{j=1}^{2} K_{j \ell}^{i}(x)\left(\partial \phi_{j} / \partial u_{1}\right)=L_{\ell}^{i}\left(x, \partial \phi_{1} / \partial u_{2}, \partial \phi_{2} / \partial u_{2}\right), \quad(1 \leq i, \ell \leq 2) .
$$

Denote by $K^{i}(x)$ the $2 \times 2$ matrix $\left(K_{j \ell}^{i}(x)\right)_{1 \leq j, \ell \leq 2}$. By an easy calculation we see that

$$
\operatorname{det} K^{i}(x)=\Lambda(x)\left(a_{2}^{i 1}(x) a_{1}^{i 2}(x)-a_{2}^{i 2}(x) a_{1}^{i 1}(x)\right)\left(a_{2}^{22}(x) a_{1}^{12}(x)-a_{2}^{12}(x) a_{1}^{22}(x)\right) .
$$

Theorem 4.1. We assume that $\operatorname{det} K^{i}\left(x_{0}\right) \neq 0$. Then the system of defining equations of $\mathfrak{x}(P)$ around $x_{0}$ is given in the following form:

$$
\begin{aligned}
& \partial \phi_{1} / \partial u_{1}=a(x)\left(\partial \phi_{1} / \partial u_{2}\right)+\partial \phi_{2} / \partial u_{2}, \\
& \partial \phi_{2} / \partial u_{1}=b(x)\left(\partial \phi_{1} / \partial u_{2}\right)
\end{aligned}
$$

where $a(x)$ and $b(x)$ can be expressed as functions of $a_{k}^{i j}(x)$.

Proof. Since $\operatorname{det} K^{i}\left(x_{0}\right) \neq 0$, (4.1) is written around $x_{0}$ by the form

$$
\partial \phi_{j} / \partial u_{1}=F_{j}^{i}\left(x, \partial \phi_{1} / \partial u_{2}, \partial \phi_{2} / \partial u_{2}\right), \quad(1 \leq i, j \leq 2)
$$

and $F_{j}^{i}$ is written by

$$
F_{j}^{i}\left(x, \partial \phi_{1} / \partial u_{2}, \partial \phi_{2} / \partial u_{2}\right)=N_{j 1}^{i}(x)\left(\partial \phi_{1} / \partial u_{2}\right)+N_{j 2}^{i}(x)\left(\partial \phi_{2} / \partial u_{2}\right)
$$

where

$$
\begin{aligned}
N_{1 \ell}^{i}(x) \operatorname{det} K^{i}(x)= & a_{2}^{i 2}(x) a_{2}^{i \ell}(x) \\
& +\Lambda(x) a_{2}^{i 2}(x) a_{1}^{i \ell}(x)\left(a_{2}^{12}(x) a_{1}^{21}(x)-a_{2}^{22}(x) a_{1}^{11}(x)\right) \\
& +\Lambda(x) a_{2}^{i \ell}(x) a_{1}^{i 2}(x)\left(a_{2}^{21}(x) a_{1}^{12}(x)-a_{2}^{11}(x) a_{1}^{22}(x)\right) \\
& +\Lambda(x) a_{1}^{i 2}(x) a_{1}^{i \ell}(x)\left(a_{2}^{11}(x) a_{2}^{22}(x)-a_{2}^{12}(x) a_{2}^{21}(x)\right) \\
-N_{2 \ell}^{i}(x) \operatorname{det} K^{i}(x)= & a_{2}^{i 1}(x) a_{2}^{i \ell}(x) \\
& +\Lambda(x) a_{2}^{i 2}(x) a_{1}^{i \ell}(x)\left(a_{2}^{12}(x) a_{1}^{21}(x)-a_{2}^{22}(x) a_{1}^{11}(x)\right) \\
& +\Lambda(x) a_{2}^{i \ell}(x) a_{1}^{11}(x)\left(a_{2}^{21}(x) a_{1}^{12}(x)-a_{2}^{11}(x) a_{1}^{22}(x)\right) \\
& +\Lambda(x) a_{1}^{i 1}(x) a_{1}^{i \ell}(x)\left(a_{2}^{11}(x) a_{2}^{22}(x)-a_{2}^{12}(x) a_{2}^{21}(x)\right)
\end{aligned}
$$

Since the identity transformation of $E$ is a solution of (4.2), we obtain 
$N_{12}^{i}(x)=1$ and $N_{22}^{i}(x)=0$. Therefore the system of defining equations of $\mathfrak{U}(P)$ at $x$ is written by

$$
\begin{aligned}
& \partial \phi_{1} / \partial u_{1}=N_{1}^{i}(x)\left(\partial \phi_{1} / \partial u_{2}\right)+\partial \phi_{2} / \partial u_{2}, \\
& \partial \phi_{2} / \partial u_{1}=N_{2}^{i}(x)\left(\partial \phi_{1} / \partial u_{2}\right), \quad(i=1,2)
\end{aligned}
$$

where $N_{1}^{i}(x)=N_{11}^{i}(x)$ and $N_{2}^{i}(x)=N_{21}^{i}(x)$.

Now we shall prove that $N_{1}^{1}(x)=N_{1}^{2}(x)$ and $N_{2}^{1}(x)=N_{2}^{2}(x)$. For the simplicity of expressions, hereafter we set $a_{k}(x)=\left(\begin{array}{ll}\alpha_{k}(x) & \beta_{k}(x) \\ \gamma_{k}(x) & \delta_{k}(x)\end{array}\right)$. Then we have

$$
\begin{aligned}
N_{1}^{1}(x) \operatorname{det} K^{1}(x)= & \Lambda(x)\left\{\beta_{2}(x) \alpha_{2}(x)\left(\alpha_{1}(x) \delta_{1}(x)-\beta_{1}(x) \gamma_{1}(x)\right)\right. \\
& +\beta_{2}(x) \alpha_{1}(x)\left(\beta_{2}(x) \gamma_{1}(x)-\delta_{2}(x) \alpha_{1}(x)\right) \\
& +\alpha_{2}(x) \beta_{1}(x)\left(\gamma_{2}(x) \beta_{1}(x)-\alpha_{2}(x) \delta_{1}(x)\right) \\
& \left.+\beta_{1}(x) \alpha_{1}(x)\left(\alpha_{2}(x) \delta_{2}(x)-\beta_{2}(x) \gamma_{2}(x)\right)\right\} \\
= & \Lambda(x)\left(\alpha_{2}(x) \beta_{1}(x)-\beta_{2}(x) \alpha_{1}(x)\right) \\
& \times\left(\gamma_{2}(x) \beta_{1}(x)-\alpha_{2}(x) \delta_{1}(x)+\alpha_{1}(x) \delta_{2}(x)-\beta_{2}(x) \gamma_{1}(x)\right) .
\end{aligned}
$$

Therefore

$$
\begin{aligned}
N_{1}^{1}(x)= & \left(\delta_{2}(x) \beta_{1}(x)-\beta_{2}(x) \delta_{1}(x)\right)^{-1} \\
& \left.\times\left(\gamma_{2}(x) \beta_{1}(x)-\alpha_{2}(x) \delta_{1}(x)\right)+\alpha_{1}(x) \delta_{2}(x)-\beta_{2}(x) \gamma_{1}(x)\right) .
\end{aligned}
$$

On the other hand

$$
\begin{aligned}
N_{1}^{2}(x) \operatorname{det} K^{2}(x)= & \Lambda(x)\left\{\delta_{2}(x) \gamma_{2}(x)\left(\alpha_{1}(x) \delta_{1}(x)-\beta_{1}(x) \gamma_{1}(x)\right)\right. \\
& +\delta_{2}(x) \gamma_{1}(x)\left(\beta_{2}(x) \gamma_{1}(x)-\delta_{2}(x) \alpha_{1}(x)\right) \\
& +\gamma_{2}(x) \delta_{1}(x)\left(\gamma_{2}(x) \beta_{1}(x)-\alpha_{2}(x) \delta_{1}(x)\right) \\
& \left.+\delta_{1}(x) \gamma_{1}(x)\left(\alpha_{2}(x) \delta_{2}(x)-\beta_{2}(x) \gamma_{2}(x)\right)\right\} \\
= & \Lambda(x)\left(\gamma_{2}(x) \delta_{1}(x)-\delta_{2}(x) \gamma_{1}(x)\right) \\
& \times\left(\gamma_{2}(x) \beta_{1}(x)-\alpha_{2}(x) \delta_{1}(x)+\alpha_{1}(x) \delta_{2}(x)-\beta_{2}(x) \gamma_{1}(x)\right)
\end{aligned}
$$

which shows

$$
\begin{aligned}
N_{1}^{2}(x)= & \left(\delta_{2}(x) \beta_{1}(x)-\beta_{2}(x) \delta_{1}(x)\right)^{-1} \\
& \times\left(\gamma_{2}(x) \beta_{1}(x)-\alpha_{2}(x) \delta_{1}(x)+\alpha_{1}(x) \delta_{2}(x)-\beta_{2}(x) \gamma_{1}(x)\right)
\end{aligned}
$$

and thus we get $N_{1}^{1}(x)=N_{1}^{2}(x)$. Similarly we can prove that

$$
N_{2}^{1}(x)=N_{2}^{2}(x)=\left(\alpha_{2}(x) \gamma_{1}(x)-\alpha_{1}(x) \gamma_{2}(x)\right)\left(\delta_{2}(x) \beta_{1}(x)-\beta_{2}(x) \delta_{1}(x)\right)^{-1}
$$

and this completes the proof of Theorem 4.1. 
Note that, if the assumptions $\alpha_{2}\left(x_{0}\right) \beta_{2}\left(x_{0}\right) \gamma_{2}\left(x_{0}\right) \delta_{2}\left(x_{0}\right) \neq 0$ and $\alpha_{1}(x) / \alpha_{2}(x)$ $=\beta_{1}(x) / \beta_{2}(x)=\gamma_{1}(x) / \gamma_{2}(x)=\delta_{1}(x) / \delta_{2}(x)$ around $x_{0}$ in Proposition 3.1 are satisfied, then $\operatorname{det} K^{i}\left(x_{0}\right)=0$. In this case we have seen that $\mathfrak{X}(P)=\mathscr{A}(E)$.

Corollary 4.2. If, in (4.2), $a(x)$ or $b(x)$ is not constant around $x_{0}$, then $\mathfrak{A}(P)$ is generated by the maximal subgroup of the affine transformation group on $R^{2}$ whose linear part is the center of $G L(2, R)$.

Proof. Since $\partial \phi_{i} / \partial u_{j}$ does not depend on $x$, from (4.2) we get $\partial \phi_{1} / \partial u_{2}$ $=\partial \phi_{2} / \partial u_{1}=0$ and $\partial \phi_{1} / \partial u_{1}=\partial \phi_{2} / \partial u_{2}$. These imply that $\phi_{1}\left(u_{1}, u_{2}\right)=\alpha u_{1}+\beta$ and $\phi_{2}\left(u_{1}, u_{2}\right)=\alpha u_{2}+\tilde{\beta}$ where $\alpha, \beta$ and $\tilde{\beta}$ are constants and $\alpha \neq 0$. This completes the proof.

Remark 4.1. If $P(x, D)$ is the Cauchy-Riemann operator, then we see that $\alpha_{1}(x)=\delta_{1}(x)=\gamma_{2}(x)=1, \beta_{2}(x)=-1$ and $\alpha_{2}(x)=\beta_{1}(x)=\gamma_{1}(x)=$ $\delta_{2}(x)=0$. Therefore it is easy to see that $K^{1}(x)=\left(\begin{array}{cc}0 & 1 \\ -1 & 0\end{array}\right)$ and $K^{2}(x)=$ $\left(\begin{array}{ll}1 & 0 \\ 0 & 1\end{array}\right)$. Thus $\operatorname{det} K^{1}(x)=\operatorname{det} K^{2}(x)=1 \neq 0$ and by Theorem 4.1 , the system of defining equations of $\mathfrak{Y}(P)$ is given in the following form:

$$
\begin{aligned}
& \partial \phi_{1} / \partial u_{1}=a(x)\left(\partial \phi_{1} / \partial u_{2}\right)+\partial \phi_{2} / \partial u_{2}, \\
& \partial \phi_{2} / \partial u_{1}=b(x)\left(\partial \phi_{1} / \partial u_{2}\right) .
\end{aligned}
$$

In the proof of Theorem 4.1, we have seen that

$$
\begin{aligned}
a(x)= & \left(\delta_{2}(x) \beta_{1}(x)-\beta_{2}(x) \delta_{1}(x)\right)^{-1} \\
& \times\left(\gamma_{2}(x) \beta_{1}(x)-\alpha_{2}(x) \delta_{1}(x)+\alpha_{1}(x) \delta_{2}(x)-\beta_{2}(x) \gamma_{1}(x)\right)
\end{aligned}
$$

and

$$
\begin{aligned}
b(x)= & \left(\delta_{2}(x) \beta_{1}(x)-\beta_{2}(x) \delta_{1}(x)\right)^{-1} \\
& \times\left(\alpha_{2}(x) \gamma_{1}(x)-\alpha_{1}(x) \gamma_{2}(x)\right) .
\end{aligned}
$$

Therefore for the Cauchy-Riemann operator $P(x, D)$ we get $a(x)=0$ and $b(x)=-1$. That is, the system of defining equations of $\mathfrak{A}(P)$ is given by

$$
\begin{aligned}
& \partial \phi_{1} / \partial u_{1}=\partial \phi_{2} / \partial u_{2}, \\
& \partial \phi_{2} / \partial u_{1}=-\partial \phi_{1} / \partial u_{2} .
\end{aligned}
$$

These are also the Cauchy-Riemann equations.

There are two ways to generalize the Cauchy-Riemann operator on $J^{1}\left(R^{2}, R^{2}\right)$.

One is the way to generalize it to the following pseudoelliptic system of differential equations on $J^{1}\left(R^{2 m}, R^{2 m}\right)$ : 


$$
P: \begin{aligned}
& \sum_{\ell=1}^{m}\left(p_{\ell 1}^{k 1}-p_{\ell 2}^{k 2}\right)=0, \\
& \sum_{\ell=1}^{m}\left(p_{\ell 1}^{k 2}+p_{\ell 2}^{k 1}\right)=0 \quad(k=1, \cdots, m),
\end{aligned}
$$

where $p_{\ell j}^{k i}=\partial u_{\imath}^{k} / \partial x_{j}^{\ell}(k, \ell=1, \cdots, m ; i, j=1,2)$.

Another way is to generalize it to the Cauchy-Riemann equations on $J^{1}\left(R^{2 m}, R^{2 m}\right)$ which is the following overdetermined pseudoelliptic system:

$$
P: \quad \begin{aligned}
& p_{\ell 1}^{k 1}-p_{\ell 2}^{k 2}=0, \\
& \\
& p_{\ell 1}^{k 2}-p_{\ell 2}^{k 1}=0, \quad(k, \ell=1, \cdots, m) .
\end{aligned}
$$

In any case, this suggests to us that it is reasonable to extend our consideration of pseudoelliptic systems of differential equations on $J^{1}\left(R^{2}, R^{2}\right)$ to pseudoelliptic systems or overdetermined pseudoelliptic systems of differential equations on $J^{1}\left(R^{2 m}, R^{2 m}\right)$.

Remark 4.2. Let us consider a following pseudoelliptic system of differential equations on $J^{1}\left(R^{n}, R^{2}\right)$ where $n \geq 3$ :

$$
P: \quad \sum_{h=1}^{2} \sum_{k=1}^{n} a_{k}^{i n}(x) p_{k}^{h}=0, \quad(i=1,2),
$$

where $p_{k}^{h}=\partial u_{h} / \partial x_{k}$. Then by similar arguments to the proof of Theorem 4.1 we can see that the system of defining equations of $\mathfrak{X}(P)$ is given by an overdetermined system in the following form under generic conditions:

$$
\begin{aligned}
& \partial \phi_{1} / \partial u_{1}=a_{(k)}(x)\left(\partial \phi_{1} / \partial u_{2}\right)+\partial \phi_{2} / \partial u_{2}, \\
& \partial \phi_{2} / \partial u_{1}=b_{(k)}(x)\left(\partial \phi_{1} / \partial u_{2}\right), \quad(k=1, \cdots, n-1) .
\end{aligned}
$$

Therefore, in this case, even if all $a_{(k)}(x)$ and $b_{(k)}(x)$ are constants, except for the case $a_{(1)}=\cdots=a_{(n-1)}$ and $b_{(1)}=\cdots=b_{(n-1)}, \mathfrak{A}(P)$ is generated by the maximal subgroup of the affine transformation group on $R^{2}$ whose linear part is the center of $G L(2, R)$.

\section{§2. Differential invariants of differential operators of infinite type}

5. Let $\Gamma$ be a pseudogroup on a manifold $M$ and denote by $\mathfrak{L}_{\Gamma}$ the sheaf of germs of local vector fields $X$ on $M$ which generate local 1parameter groups of local transformations $\phi_{t}^{X}$ belonging to $\Gamma$. This sheaf $\mathfrak{\Omega}_{\Gamma}$ is a weak Lie algebra sheaf ([2]).

Let us consider the $k$-jet space $J^{k}(N, M), k \geq 1$, whose element is a $k$-jet $j_{x}^{k}(f)$ of a local map $f$ of $N$ to $M$, where $x$ belongs to $N$, called the 
source of $p=j_{x}^{k}(f)$ and denoted by $\alpha^{k}(p)$. Furthermore we set $\beta^{k}(p)=$ $f(x)$ and $J^{0}(N, M)=N \times M$.

It is easy to see that any pseudogroup $\Gamma$ on $M$ is naturally lifted to a pseudogroup $\Gamma^{(k)}$ on $J^{k}(N, M)$ and so the sheaf $\mathfrak{L}_{\Gamma}$ is also lifted to a weak Lie algebra sheaf $\mathfrak{\Omega}_{\Gamma}^{(k)}$ on $J^{k}(N, M) . \Gamma^{(0)}$ (resp. $\mathfrak{L}_{\Gamma}^{(0)}$ ) is considered as a pseudogroup (resp. a weak Lie algebra sheaf) $1 \times \Gamma$ (resp. $0 \times \mathbb{2}_{\Gamma}$ ) on $N \times M$.

Denote by $\left(\Omega_{T}^{(k)}\right)_{p}^{0}$ the isotropy algebra of the stalk $\left(\Omega_{T}^{(k)}\right)_{p}$ over $p$. Then $\left(D_{\Gamma}^{(k)}\right)_{p}=\left(\Omega_{\Gamma}^{(k)}\right)_{p} /\left(\Omega_{\Gamma}^{(k)}\right)_{p}^{0}$ is regarded as a subspace of the tangent space $T_{p}$ of $J^{k}(N, M)$ at $p$.

Definition 5.1. $\Gamma$ is called $(N, k)$-regular if, for each integer $\ell$, $0 \leq \ell \leq k, \operatorname{dim}\left(D_{\Gamma}^{(\ell)}\right)_{p}$ is constant.

Assume that $\Gamma$ is $(N, k)$-regular. Then the correspondence $D_{\Gamma}^{(k)}$ : $J^{k}(N, M) \ni p \rightarrow\left(D_{\Gamma}^{(k)}\right)_{p} \subset T_{p}$ is an involutive distribution on $J^{k}(N, M)$.

Definition 5.2. A function $y$ given on a small neighbourhood $\mathfrak{U}$ of $p \in J^{k}(N, M)$ is called a differential invariant of a vector field $Z$ on $\mathfrak{U}$ if $Z y=0$. If, for any cross-section $Z$ of $D_{\Gamma}^{(k)}$ on $\mathfrak{U}, y$ is a differential invariant of $Z$, then $y$ is called a differential invariant of $\Gamma$. Furthermore if $\left\{y_{1}, \cdots, y_{q}\right\}, q=\operatorname{codim} D_{\Gamma}^{(k)}$, is a family of functionally independent differential invariants of $\Gamma$ on $\mathfrak{H}$, it is called a fundamental system of differential invariants of $\Gamma$ on $\mathfrak{U}$.

6. In the following we assume that $N=M=R^{2}$ and let us consider a differential operator $P(x, D)$ of order 1 satisfying the following conditions:

(1) $P(x, D)$ is pseudoelliptic at $x_{0} \in R^{2}$.

(2) $P(x, D)$ is pseudoinvolutive at any $p \in I(P)$.

(3) $\operatorname{det} K^{i}\left(x_{0}\right) \neq 0, i=1,2$.

(4) $P(x, D)=P_{\langle 1\rangle}(x, D)$.

Then by Theorem 4.1 the system of defining equations of the automorphism pseudogroup $\mathfrak{A}(P)$ is given by

$$
\begin{aligned}
& \partial \phi_{1} / \partial u_{1}=a(x)\left(\partial \phi_{1} / \partial u_{2}\right)+\partial \phi_{2} / \partial u_{2}, \\
& \partial \phi_{2} / \partial u_{1}=b(x)\left(\partial \phi_{1} / \partial u_{2}\right) .
\end{aligned}
$$

DeFinition 6.1. Let $P(x, D)$ be a differential operator satisfying the conditions (1), (2), (3) and (4). Then $P(x, D)$ or a system of differential 
equations $P: P(x, D) u=0$ is said to be of infinite type at $x_{0}$ if $a(x)$ and $b(x)$ are constants around $x_{c}$. The vector $(a, b)$ is called the structure vector of $P(x, D)$ or $P$.

Assume that $P(x, D)$ is of infinite type at $x_{0}$ with the structure vector $(a, b)$. By considering (6.1) infinitesimally, a vector field $X\left(u_{1}, u_{2}\right)\left(\partial / \partial u_{1}\right)+$ $Y\left(u_{1}, u_{2}\right)\left(\partial / \partial u_{2}\right)$ defined on $\mathfrak{U} \subset M=R^{2}$ is a local cross-section of $\mathfrak{L}_{\mathscr{U}(P)}$ if and only if it satisfies

$$
\begin{aligned}
& \partial X / \partial u_{1}=a\left(\partial X / \partial u_{2}\right)+\partial Y / \partial u_{2}, \\
& \partial Y / \partial u_{1}=b\left(\partial X / \partial u_{2}\right)
\end{aligned}
$$

or simply (6.2) is written by

$$
\begin{aligned}
& X_{u_{1}}=a X_{u_{2}}+Y_{u_{2}}, \\
& Y_{u_{1}}=b X_{u_{2}}
\end{aligned}
$$

Remark 6.1. If $P(x, D)$ is of infinite type at $x_{0}$ with the structure vector $(a, b)$, then, as is calculated in the proof of Theorem 4.1, the constants $a$ and $b$ are expressed around $x_{0}$ as

$$
\begin{aligned}
a= & \left(\delta_{2}(x) \beta_{1}(x)-\beta_{2}(x) \delta_{1}(x)\right)^{-1} \\
& \times\left(\gamma_{2}(x) \beta_{1}(x)-\alpha_{2}(x) \delta_{1}(x)+\alpha_{1}(x) \delta_{2}(x)-\beta_{2}(x) \gamma_{1}(x)\right)
\end{aligned}
$$

and

$$
b=\left(\delta_{2}(x) \beta_{1}(x)-\beta_{2}(x) \delta_{1}(x)\right)^{-1}\left(\alpha_{2}(x) \gamma_{1}(x)-\alpha_{1}(x) \gamma_{2}(x)\right) .
$$

Let $W$ be a cross-section of $\mathfrak{L}_{\mathfrak{A}(P)}$ defined on $\mathfrak{U}$. Then $W$ is naturally lifted to a vector field $W^{(1)}$ on $\mathfrak{U}^{(1)}=\left(\beta^{1}\right)^{-1}(\mathfrak{H})$ which is a cross-section of $\mathfrak{L}_{\mathfrak{Q}(P)}^{(1)}$. Consider the canonical coordinate system $\left\{x_{1}, x_{2}, u_{1}, u_{2}, p_{1}^{1}, p_{1}^{2}, p_{2}^{1}, p_{2}^{2}\right\}$ on $J^{1}\left(R^{2}, R^{2}\right)$ introduced by $p_{j}^{i}=\partial u_{i} / \partial x_{j}$.

LEMMA 6.1. For a vector field $W=X\left(u_{1}, u_{2}\right)\left(\partial / \partial u_{1}\right)+Y\left(u_{1}, u_{2}\right)\left(\partial / \partial u_{2}\right)$, $W^{(1)}$ is expressed by

$$
\begin{aligned}
W^{(1)}= & X\left(\partial / \partial u_{1}\right)+Y\left(\partial / \partial u_{2}\right) \\
& +X_{u_{2}}\left\{\left(a p_{1}^{1}+p_{1}^{2}\right)\left(\partial / \partial p_{1}^{1}\right)+b p_{1}^{1}\left(\partial / \partial p_{1}^{2}\right)+\left(a p_{2}^{1}+p_{2}^{2}\right)\left(\partial / \partial p_{2}^{1}\right)+b p_{2}^{1}\left(\partial / \partial p_{2}^{2}\right)\right\} \\
& +Y_{u_{2}}\left\{p_{1}^{1}\left(\partial / \partial p_{1}^{1}\right)+p_{1}^{2}\left(\partial / \partial p_{1}^{2}\right)+p_{2}^{1}\left(\partial / \partial p_{2}^{1}\right)+p_{2}^{2}\left(\partial / \partial p_{2}^{2}\right)\right\} .
\end{aligned}
$$

Proof. We set $\partial_{i}^{\#}=\partial / \partial x_{i}+p_{i}^{1}\left(\partial / \partial u_{1}\right)+p_{\imath}^{2}\left(\partial / \partial u_{2}\right)$. Then we have $W^{(1)} \partial_{i}^{\#}=$ $\partial_{i}^{\sharp} W$, ([3]). Applying this equality to the function $u_{1}$ (resp. $u_{2}$ ), we get $W^{(1)} p_{1}^{1}=\partial_{i}^{\sharp} X$ (resp. $W^{(1)} p_{\imath}^{2}=\partial_{i}^{\sharp} Y$ ). This means that $W^{(1)}$ is expressed by 
$W^{(1)}=X\left(\partial / \partial u_{1}\right)+Y\left(\partial / \partial u_{2}\right)+\left(X_{u_{1}} p_{1}^{1}+X_{u_{2}} p_{1}^{2}\right)\left(\partial / \partial p_{1}^{1}\right)+\left(Y_{u_{1}} p_{1}^{1}+Y_{u_{2}} p_{1}^{2}\right)\left(\partial / \partial p_{1}^{2}\right)+$ $\left(X_{u_{1}} p_{2}^{1}+X_{u_{2}} p_{2}^{2}\right)\left(\partial / \partial p_{2}^{1}\right)+\left(Y_{u_{1}} p_{2}^{1}+Y_{u_{2}} p_{2}^{2}\right)\left(\partial / \partial p_{2}^{2}\right)$. Since $W$ satisfies (6.2), we get

$$
\begin{aligned}
W^{(1)}= & X\left(\partial / \partial u_{1}\right)+Y\left(\partial / \partial u_{2}\right) \\
& +X_{u_{2}}\left\{\left(a p_{1}^{1}+p_{1}^{2}\right)\left(\partial / \partial p_{1}^{1}\right)+b p_{1}^{1}\left(\partial / \partial p_{1}^{2}\right)+\left(a p_{2}^{1}+p_{2}^{2}\right)\left(\partial / \partial p_{2}^{1}\right)+b p_{2}^{1}\left(\partial / \partial p_{2}^{2}\right)\right\} \\
& +Y_{u_{2}}\left\{p_{1}^{1}\left(\partial / \partial p_{1}^{1}\right)+p_{1}^{2}\left(\partial / \partial p_{1}^{2}\right)+p_{2}^{1}\left(\partial / \partial p_{2}^{1}\right)+p_{2}^{2}\left(\partial / \partial p_{2}^{2}\right)\right\} .
\end{aligned}
$$

This completes the proof.

Proposition 6.2. Assume that $P(x, D)$ is of infinite type at $x_{0}$ with the structure vector $(a, b)$. Then a function $f$ given on a neighbourhood $\mathfrak{H}$ of $p \in J^{1}\left(R^{2}, R^{2}\right)$ with $\alpha^{1}(p)=x_{0}$ is a differential invariant of $\mathfrak{X}(P)$ if and only if $f$ satisfies the following relations:

$$
\left\{\begin{array}{l}
\left(a p_{1}^{1}+p_{1}^{2}\right)\left(\partial f / \partial p_{1}^{1}\right)+b p_{1}^{1}\left(\partial f / \partial p_{1}^{2}\right)+\left(a p_{2}^{1}+p_{2}^{2}\right)\left(\partial f / \partial p_{2}^{1}\right)+b p_{2}^{1}\left(\partial f / \partial p_{2}^{2}\right)=0 \\
p_{1}^{1}\left(\partial f / \partial p_{1}^{1}\right)+p_{1}^{2}\left(\partial f / \partial p_{1}^{2}\right)+p_{2}^{1}\left(\partial f / \partial p_{2}^{1}\right)+p_{2}^{2}\left(\partial f / \partial p_{2}^{2}\right)=0 \\
\partial f / \partial u_{1}=0 \text { and } \partial f / \partial u_{2}=0
\end{array}\right.
$$

Proof. Since, for any constants $\beta, \beta^{\prime}, \gamma$ and $\gamma^{\prime}$, there exist constants $\alpha$ and $\alpha^{\prime}$ such that $(X, Y)$, where $X=\alpha u_{1}+\beta u_{2}+\gamma$ and $Y=\alpha^{\prime} u_{1}+\beta^{\prime} u_{2}$ $+\gamma^{\prime}$, is a solution of (6.2). Therefore at each point $u=\left(u_{1}, u_{2}\right)$ we have $\left\{\left(X(u), Y(u), X_{u_{2}}(u), Y_{u_{2}}(u)\right) ; X\left(\partial / \partial u_{1}\right)+Y\left(\partial / \partial u_{2}\right)\right.$ is a cross-section of $\mathfrak{Q}_{\mathfrak{x}(P)}$ around $u\}=R^{4}$. Then by Lemma $6.1, f$ is a differential invariant of $\mathfrak{U}(P)$ if and only if $F$ satisfies (6.3). This completes the proof of Proposition 6.2 .

7. We set $z_{1}=p_{1}^{1}, z_{2}=p_{1}^{2}, z_{3}=p_{2}^{1}$ and $z_{4}=p_{2}^{2}$ and let us consider differential invariants of a vector field $Z=\left(a z_{1}+z_{2}\right)\left(\partial / \partial z_{1}\right)+b z_{1}\left(\partial / \partial z_{2}\right)+$ $\left(a z_{3}+z_{4}\right)\left(\partial / \partial z_{3}\right)+b z_{3}\left(\partial / \partial z_{4}\right)$ on $J^{1}\left(R^{2}, R^{2}\right)$. If we regard $Z$ as a vector field on $R^{4}$ with the coordinate system $\left\{z_{1}, z_{2}, z_{3}, z_{4}\right\}$ and find a fundamental system of first integrals of $Z$ around $z=\left(p_{1}^{1}(p), p_{1}^{2}(p), p_{2}^{1}(p), p_{2}^{2}(p)\right) \in R^{4}$, we can get a fundamental system of differential invariants of $Z$ around $p=$ $j_{x_{0}}^{1}(f)$ because the functions $x_{1}, x_{2}, u_{1}$ and $u_{2}$ are differential invariants of $Z$ around $p$.

It is well-known that there exists a local coordinate system $\left\{y_{1}, y_{2}\right.$, $\left.y_{3}, y_{4}\right\}$ on a neighbourhood $\mathfrak{U}$ of the origin $0 \in R^{4}$ such that $Z=\partial / \partial y_{1}$ on U. If we can find such a coordinate system, then $\left\{y_{2}, y_{3}, y_{4}\right\}$ is a fundamental system of first integrals of $Z$ on $\mathfrak{U}$. This coordinate system $\left\{y_{1}, y_{2}, y_{3}, y_{4}\right\}$ can be found by solving the following system of ordinary differential equations 


$$
\left\{\begin{array}{l}
d z_{1} / d t=a z_{1}+z_{2} \\
d z_{2} / d t=b z_{1} \\
d z_{3} / d t=a z_{3}+z_{4} \\
d z_{4} / d t=b z_{3}
\end{array}\right.
$$

with the initial condition $z_{1}(0)=0, z_{2}(0)=y_{2}, z_{3}(0)=y_{3}, z_{4}(0)=y_{4}$ and by setting $y_{1}=t([1])$.

Lemma 7.1. Assume that $a^{2}+4 b>0$ and set $\rho_{1}=\left(a+\sqrt{a^{2}+4 b}\right) / 2$, $\rho_{2}=\left(a-\sqrt{a^{2}+4 b}\right) / 2$ and $H=\left(\rho_{1} z_{1}+z_{2}\right) /\left(\rho_{2} z_{1}+z_{2}\right)$. Then around a point $z=\left(z_{1}, z_{2}, z_{3}, z_{4}\right)$ with $\left(\rho_{1} z_{1}+z_{2}\right)\left(\rho_{2} z_{1}+z_{2}\right) \neq 0$, the following family of functions $\left\{f_{1}, f_{2}, f_{3}\right\}$ is a fundamental system of first integrals of $Z$ defined as above:

$$
\begin{aligned}
& f_{1}=\left(\rho_{1}-\rho_{2}\right) z_{1}\left(H^{\rho_{1}\left(\rho_{1}-\rho_{2}\right)-1}-H^{\rho_{2}\left(\rho_{1}-\rho_{2}\right)-1}\right)^{-1} \\
& f_{2}=\left(\rho_{2} z_{3}+z_{4}\right)\left(\left(\rho_{2}-\rho_{1}\right) H^{\rho_{2}\left(\rho_{1}-\rho_{2}\right)-1}\right)^{-1}-\left(\rho_{1} z_{3}+z_{4}\right)\left(\left(\rho_{2}-\rho_{1}\right) H^{\rho_{1}\left(\rho_{1}-\rho_{2}\right)-1}\right)^{-1}, \\
& f_{3}=\rho_{2}\left(\rho_{1} z_{3}+z_{4}\right)\left(\left(\rho_{2}-\rho_{1}\right) H^{\rho_{1}\left(\rho_{1}-\rho_{2}\right)-1}\right)^{-1}-\rho_{1}\left(\rho_{2} z_{3}+z_{4}\right)\left(\left(\rho_{2}-\rho_{1}\right) H^{\rho_{2}\left(\rho_{1}-\rho_{2}\right)-1}\right)^{-1} .
\end{aligned}
$$

Proof. Associated with the system of ordinary differential equations (7.1), we have the following algebraic equation with respect to $\rho$ :

$$
\left|\begin{array}{cccc}
a-\rho & 1 & 0 & 0 \\
b & -\rho & 0 & 0 \\
0 & 0 & a-\rho & 1 \\
0 & 0 & b & -\rho
\end{array}\right|=\left(\rho^{2}-a \rho-b\right)^{2}=0
$$

According to the theory of ordinary differential equations, in the case $a^{2}+4 b>0$, we can find the following unique solution $\left(z_{1}(t), z_{2}(t), z_{3}(t), z_{4}(t)\right)$ of $(7.1)$ with the initial condition $z_{1}(0)=0, z_{2}(0)=y_{2}, z_{3}(0)=y_{3}, z_{4}(0)=y_{4}$;

$$
\left\{\begin{array}{l}
z_{1}(t)=\left(\rho_{1}-\rho_{2}\right)^{-1} y_{2}\left(e^{\rho_{1} t}-e^{\rho_{2} t}\right), \\
z_{2}(t)=\left(\rho_{1}-\rho_{2}\right)^{-1} y_{2}\left(\rho_{1} e^{\rho_{2} t}-\rho_{2} e^{\rho_{1} t}\right), \\
z_{3}(t)=\left(\rho_{1}-\rho_{2}\right)^{-1}\left\{\left(\rho_{1} y_{3}+y_{4}\right) e^{\rho_{1} t}-\left(\rho_{2} y_{3}+y_{4}\right) e^{\rho_{2} t}\right\}, \\
z_{4}(t)=\left(\rho_{1}-\rho_{2}\right)^{-1}\left\{\left(\rho_{2} y_{3}+y_{4}\right) \rho_{1} e^{\rho_{2} t}-\left(\rho_{1} y_{3}+y_{4}\right) \rho_{2} e^{\rho_{1} t}\right\} .
\end{array}\right.
$$

Setting $y_{1}=t$ in the right hand side of (7.2) and solving (7.2) with respect to $\left\{y_{1}, y_{2}, y_{3}, y_{4}\right\}$, we get

$$
\begin{aligned}
& y_{1}=\left(\rho_{1}-\rho_{2}\right)^{-1} \log \left(\left(\rho_{1} z_{1}+z_{2}\right)\left(\rho_{2} z_{1}+z_{2}\right)^{-1}\right), \\
& y_{2}=\left(\rho_{1}-\rho_{2}\right) z_{1}\left(H^{\rho_{1}\left(\rho_{1}-\rho_{2}\right)-1}-H^{\rho_{2}\left(\rho_{1}-\rho_{2}\right)-1}\right)^{-1},
\end{aligned}
$$




$$
\begin{aligned}
& y_{3}=\left(\rho_{2} z_{3}+z_{4}\right)\left(\left(\rho_{2}-\rho_{1}\right) H^{\rho_{2}\left(\rho_{1}-\rho_{2}\right)-1}\right)^{-1}-\left(\rho_{1} z_{3}+z_{4}\right)\left(\left(\rho_{2}-\rho_{1}\right) H^{\rho_{1}\left(\rho_{1}-\rho_{2}\right)-1}\right)^{-1} \\
& y_{4}=\rho_{2}\left(\rho_{1} z_{3}+z_{4}\right)\left(\left(\rho_{2}-\rho_{1}\right) H^{\rho_{1}\left(\rho_{1}-\rho_{2}\right)-1}\right)^{-1}-\rho_{1}\left(\rho_{2} z_{3}+z_{4}\right)\left(\left(\rho_{2}-\rho_{1}\right) H^{\rho_{2}\left(\rho_{1}-\rho_{2}\right)-1}\right)^{-1}
\end{aligned}
$$

which prove Lemma 7.1.

Lemma 7.2. Assume that $a^{2}+4 b<0$. Then the following family of functions $\left\{g_{1}, g_{2}, g_{3}\right\}$ is a fundamental system of first integrals of $Z$ around $a$ point $z$ with $z_{1} \neq 0$ : By setting $\theta=\sqrt{-a^{2}-4 b} / 2$ and $y_{1}=\theta^{-1} \operatorname{Cot}^{-1}\left(z_{2}\left(\theta z_{1}\right)^{-1}\right.$ $\left.+a(2 \theta)^{-1}\right)$

$$
\begin{aligned}
& g_{1}=\theta z_{1}\left(e^{\left(a y_{1}\right) / 2} \sin \left(\theta y_{1}\right)\right)^{-1} \\
& g_{2}=e^{-\left(a y_{1}\right) / 2}\left(\left(\cos \left(\theta y_{1}\right)-a(2 \theta)^{-1} \sin \left(\theta y_{1}\right)\right) z_{3}-\theta^{-1}\left(\sin \left(\theta y_{1}\right)\right) z_{4}\right), \\
& g_{3}=e^{-\left(a y_{1}\right) / 2}\left(\left(\cos \left(\theta y_{1}\right)+a(2 \theta)^{-1} \sin \left(\theta y_{1}\right)\right) z_{4}+(4 \theta)^{-1}\left(4 \theta^{2}+a^{2}\right)\left(\sin \left(\theta y_{1}\right)\right) z_{3}\right) .
\end{aligned}
$$

Proof. In the case $a^{2}+4 b<0$, we have the following unique solution $\left(z_{1}(t), z_{2}(t), z_{3}(t), z_{4}(t)\right)$ of $(7.1)$ with the initial condition $z_{1}(0)=0$, $z_{2}(0)=y_{2}, z_{3}(0)=y_{3}, z_{4}(0)=y_{4}$ :

$$
\left\{\begin{aligned}
z_{1}(t)= & \theta^{-1} y_{2} e^{(a t) / 2} \sin (\theta t) \\
z_{2}(t)= & (2 \theta)^{-1} y_{2} e^{(a t) / 2}(2 \theta \cos (\theta t)-a \sin (\theta t)) \\
z_{3}(t)= & e^{(a t) / 2}\left(y_{3} \cos (\theta t)+(2 \theta)^{-1}\left(a y_{3}+2 y_{4}\right) \sin (\theta t)\right) \\
z_{4}(t)= & (4 \theta)^{-1}\left(a y_{3}+2 y_{4}\right) e^{(a t) / 2}(2 \theta \cos (\theta t)-a \sin (\theta t)) \\
& -2^{-1} y_{3} e^{(a t) / 2}(a \cos (\theta t)+2 \theta \sin (\theta t))
\end{aligned}\right.
$$

By setting $y_{1}=t$ in the right hand side of (7.4), let us solve (7.4) with respect to $\left\{y_{1}, y_{2}, y_{3}, y_{4}\right\}$.

From the 1-st and 2-nd relations of (7.4), we get

$$
\begin{aligned}
z_{2}\left(z_{1}\right)^{-1} & =\left(\theta \cos \left(\theta y_{1}\right)-(a / 2) \sin \left(\theta y_{1}\right)\right)\left(\sin \left(\theta y_{1}\right)\right)^{-1} \\
& =\theta \cot \left(\theta y_{1}\right)-a / 2
\end{aligned}
$$

and so

$$
y_{1}=\theta^{-1} \operatorname{Cot}^{-1}\left(z_{2}\left(\theta z_{1}\right)^{-1}+a(2 \theta)^{-1}\right) .
$$

Then by an easy calculation we obtain

$$
\left\{\begin{aligned}
y_{2}= & \theta z_{1}\left(e^{\left(a y_{1}\right) / 2} \sin \left(\theta y_{1}\right)\right)^{-1}, \\
y_{3}= & e^{-\left(a y_{1}\right) / 2}\left(\left(\cos \left(\theta y_{1}\right)-a(2 \theta)^{-1} \sin \left(\theta y_{1}\right)\right) z_{3}-\theta^{-1}\left(\sin \left(\theta y_{1}\right)\right) z_{4}\right), \\
y_{4}= & e^{-\left(a y_{1}\right) / 2}\left(\left(\cos \left(\theta y_{1}\right)+a(2 \theta)^{-1} \sin \left(\theta y_{1}\right)\right) z_{4}\right. \\
& \left.+(4 \theta)^{-1}\left(4 \theta^{2}+a^{2}\right)\left(\sin \left(\theta y_{1}\right)\right) z_{3}\right) .
\end{aligned}\right.
$$

This completes the proof. 
LEmma 7.3. Assume that $a^{2}+4 b=0$. Then around a point $z$ with $a z_{1}+2 z_{2} \neq 0$, the following family of functions $\left\{h_{1}, h_{2}, h_{3}\right\}$ is a fundamental system of first iategrals of $Z$ : By setting $y_{1}=2 z_{1}\left(a z_{1}+2 z_{2}\right)^{-1}$,

$$
\begin{aligned}
& h_{1}=\left(y_{1}\right)^{-1} z_{1} e^{-\left(a y_{1}\right) / 2}, \\
& h_{2}=\left(\left(1-(a / 2) y_{1}\right) z_{3}-y_{1} z_{4}\right) e^{-\left(a y_{1}\right) / 2}, \\
& h_{3}=\left((1+(a / 2)) y_{1} z_{4}+\left(a^{2} / 4\right) y_{1} z_{3}\right) e^{-\left(a y_{1}\right) / 2} .
\end{aligned}
$$

Proof. The unique solution $\left(z_{1}(t), z_{2}(t), z_{3}(t), z_{4}(t)\right)$ of (7.1) with the initial condition $z_{1}(0)=0, z_{2}(0)=y_{2}, z_{3}(0)=y_{3}, z_{4}(0)=y_{4}$ is as follows:

$$
\left\{\begin{aligned}
z_{1}(t)= & -y_{2} e^{(a t) / 2}+y_{2}(t+1) e^{(a t) / 2} \\
z_{2}(t)= & (a / 2) y_{2} e^{(a t) / 2}+y_{2}(1-(a / 2)(t+1)) e^{(a t) / 2} \\
z_{3}(t)= & \left((1-(a / 2)) y_{3}-y_{4}\right) e^{(a t) / 2}+\left((a / 2) y_{3}+y_{4}\right)(t+1) e^{(a t) / 2}, \\
z_{4}(t)= & -(a / 2)\left((1-(a / 2)) y_{3}-y_{4}\right) e^{(a t) / 2} \\
& +\left((a / 2) y_{3}+y_{4}\right)(1-(a / 2)(t+1)) e^{(a t) / 2}
\end{aligned}\right.
$$

Setting $y_{1}=t$ in the right hand side of (7.7) and solving (7.7) with respect to $\left\{y_{1}, y_{2}, y_{3}, y_{4}\right\}$, we get

$$
\begin{aligned}
& y_{1}=2 z_{1}\left(a z_{1}+2 z_{2}\right)^{-1}, \\
& y_{2}=\left(y_{1}\right)^{-1} z_{1} e^{-\left(a y_{1}\right) / 2}, \\
& y_{3}=\left(\left(1-(a / 2) y_{1}\right) z_{3}-y_{1} z_{4}\right) e^{-\left(a y_{1}\right) / 2}, \\
& y_{4}=\left((1+(a / 2)) y_{1} z_{4}+\left(a^{2} / 4\right) y_{1} z_{3}\right) e^{-\left(a y_{1}\right) / 2} .
\end{aligned}
$$

This completes the proof.

8. Let us write $z_{2}=z_{2} / z_{1}, \partial_{3}=z_{3} / z_{1}$ and $z_{4}=z_{4} / z_{1}$.

Proposition 8.1. Let $P(x, D)$ be a differential operator of infinite type at $x_{0}$ with the structure vector $(a, b)$. Then the following family of functions $\left\{x_{1}, x_{2}, I_{1}^{a, b}, I_{2}^{a, b}\right\}$ is a fundamental system of differential invariants of $\mathfrak{U}(P)$ around a point $p \in J^{1}\left(R^{2}, R^{2}\right)$ which satisfies $\left(\vec{\jmath}_{2}\right)^{2}(p)+a \vec{\jmath}_{2}(p)-b \neq 0$ :

$$
\begin{aligned}
& I_{1}^{a, b}=\left(\partial_{2} \jmath_{3}-\jmath_{4}\right) /\left(\left(\jmath_{2}\right)^{2}+a_{\jmath_{2}}-b\right), \\
& I_{2}^{a, b}=\left(\left(a+\jmath_{2}\right) \jmath_{4}-b \jmath_{3}\right) /\left(\left(\partial_{2}\right)^{2}+a_{\jmath_{2}}-b\right) .
\end{aligned}
$$

Proof. Assume that $a^{2}+4 b>0$. Then by Lemma 7.1, $f_{2} / f_{1}$ and $f_{3} / f_{1}$ are functionally independent differential invariants of $Z$ around $p$ which satisfies $\left(\rho_{1} z_{1}(p)+z_{2}(p)\right)\left(\rho_{2} z_{1}(p)+z_{2}(p)\right) \neq 0$. Since $\rho_{1}=\left(a+\sqrt{a^{2}+4 b}\right) / 2$ and $\rho_{2}=\left(a-\sqrt{a^{2}+4 b}\right) / 2,\left(\rho_{1} z_{1}(p)+z_{2}(p)\right)\left(\rho_{2} z_{1}(p)+z_{2}(p)\right) \neq 0$ if and only if $\left(\left(z_{2}\right)^{2}+a_{z_{2}}-b\right)(p) \neq 0$. 
Furthermore $\jmath_{2}=p_{1}^{2} / p_{1}^{1}, z_{3}=p_{2}^{1} / p_{1}^{1}$ and $z_{4}=p_{2}^{2} / p_{1}^{1}$ are differential invariants of the vector field $Z^{\prime}=p_{1}^{1}\left(\partial / \partial p_{1}^{1}\right)+p_{1}^{2}\left(\partial / \partial p_{1}^{2}\right)+p_{2}^{1}\left(\partial / \partial p_{2}^{1}\right)+p_{2}^{2}\left(\partial / \partial p_{2}^{2}\right)$. Since $\partial\left(f_{2} / f_{1}\right) / \partial u_{1}=\partial\left(f_{3} / f_{1}\right) / \partial u_{2}=0$, by Proposition 6.2 we see that $f_{2} / f_{1}$ and $f_{3} / f_{1}$ are differential invariants of $\mathfrak{U}(P)$. It is easy to check that

$$
\begin{aligned}
& f_{2} / f_{1}=\left(\partial_{2} z_{3}-\jmath_{4}\right) /\left(\left(\partial_{2}\right)^{2}+a_{\partial_{2}}-b\right), \\
& f_{3} / f_{1}=\left(\left(a+\jmath_{2}\right) z_{4}-b z_{3}\right) /\left(\left(\partial_{2}\right)^{2}+a_{\partial_{2}}-b\right) .
\end{aligned}
$$

Because codim $D_{2(P)}^{(1)}=4$ by Proposition 6.2, the family of functions $\left\{x_{1}, x_{2}\right.$, $\left.f_{2} / f_{1}, f_{3} \mid f_{1}\right\}$ is a fundamental system of differential invariants of $\mathfrak{X}(P)$ around $p$.

Secondly assume that $a^{2}+4 b<0$. Then by Lemma $7.2, g_{2} / g_{1}$ and $g_{3} / g_{1}$ are functionally independent differential invariants of $Z$. Note that in this case $\left(z_{2}\right)^{2}+a_{z_{2}}-b>0$. By similar arguments stated above, the family of functions $\left\{x_{1}, x_{2}, g_{2} / g_{1}, g_{3} / g_{1}\right\}$ is a fundamental system of differential invariants of $\mathscr{A}(P)$. We see that, by setting $\eta=\operatorname{Cot}^{-1}\left(\theta^{-1} z_{2}+a(2 \theta)^{-1}\right)$,

$$
\begin{aligned}
g_{2} / g_{1} & =\theta^{-1}\left(\sin \eta \cos \eta-a(2 \theta)^{-1} \sin ^{2} \eta\right) \partial_{3}-\theta^{-2}\left(\sin ^{2} \eta\right) z_{4} \\
& =\theta^{-2}\left(\sin ^{2} \eta\right)\left(\partial_{2} \partial_{3}-z_{4}\right), \\
g_{3} / g_{1} & =\theta^{-1}\left(\sin \eta \cos \eta+a(2 \theta)^{-1} \sin ^{2} \eta\right) z_{4}+\left(4 \theta^{2}\right)^{-1}\left(4 \theta^{2}+a^{2}\right)\left(\sin ^{2} \eta\right) z_{3} \\
& =\theta^{-2}\left(\sin ^{2} \eta\right)\left(\left(a+\partial_{2}\right) z_{4}+4^{-1}\left(4 \theta^{2}+a^{2}\right) \partial_{3}\right) .
\end{aligned}
$$

Since $\sin ^{2}\left(\operatorname{Cot}^{-1} x\right)=\left(1+x^{2}\right)^{-1}$, we can easily see that

$$
\theta^{-2} \sin ^{2} \eta=\left(\left(z_{2}\right)^{2}+a_{\partial_{2}}-b\right)^{-1}
$$

and we get

$$
\begin{aligned}
& g_{2} / g_{1}=\left(\hat{\partial}_{2} \vec{\partial}_{3}-\vec{\partial}_{4}\right) /\left(\left(\hat{\partial}_{2}\right)^{2}+a_{\partial_{2}}-b\right) \\
& g_{3} / g_{1}=\left(\left(a+\partial_{z_{2}}\right)_{\partial_{4}}-b b_{3}\right) /\left(\left(\partial_{2}\right)^{2}+a_{\partial_{2}}-b\right) \text {. }
\end{aligned}
$$

Finally assume that $a^{2}+4 b=0$. Then by Lemma $7.3, h_{2} / h_{1}$ and $h_{3} / h_{1}$ are functionally independent differential invariants of $Z$ around $p$ which satisfies $a z_{1}(p)+2 z_{2}(p) \neq 0$. Since $\left(\jmath_{2}\right)^{2}+a z_{2}-b=\left(z_{2}+a / 2\right)^{2}, a z_{1}(p)+$ $2 z_{2}(p) \neq 0$ if and only if $\left(\left(\xi_{2}\right)^{2}+a{\jmath_{2}}_{2}-b\right)(p) \neq 0$. Then the same arguments lead us to the assertion that the family of functions $\left\{x_{1}, x_{2}, h_{2} / h_{1}\right.$, $\left.h_{3} / h_{1}\right\}$ is a fundamental system of differential invariants of $\mathfrak{Y}(P)$ around $p$. It is easy to check that

$$
\begin{aligned}
& h_{2} / h_{1}=\left(\partial_{2} \partial_{3}-\partial_{4}\right) /\left(\partial_{2}+a / 2\right)^{2} \\
& =\left(\vec{\partial}_{2 \partial_{3}}-\vec{\jmath}_{4}\right) /\left(\left(\vec{\partial}_{2}\right)^{2}+a_{\partial_{2}}-b\right), \\
& h_{3} / h_{1}=\left(\left(a+\jmath_{2}\right)_{\partial_{4}}+\left(a^{2} / 4\right)_{\partial_{3}}\right) /\left(\partial_{2}+a / 2\right)^{2} \\
& =\left(\left(a+\jmath_{2}\right)_{z_{4}}-b_{z_{3}}\right) /\left(\left(\partial_{2}\right)^{2}+a_{\partial_{2}}-b\right) .
\end{aligned}
$$

This completes the proof of Proposition 8.1. 


\section{§3. Invariant structures of differential operators of infinite type}

9. Let $P(x, D)$ be a differential operator of infinite type at $x_{0}$ with the structure vector $(a, b)$. The system of differential equations $P(x, D) u$ $=0$ is expressed on $J^{1}\left(R^{2}, R^{2}\right)$ by

$$
\begin{aligned}
& \alpha_{1}(x) p_{1}^{1}+\beta_{1}(x) p_{1}^{2}+\alpha_{2}(x) p_{2}^{1}+\beta_{2}(x) p_{2}^{2}=0, \\
& \gamma_{1}(x) p_{1}^{1}+\delta_{1}(x) p_{1}^{2}+\gamma_{2}(x) p_{2}^{1}+\delta_{2}(x) p_{2}^{2}=0
\end{aligned}
$$

and, around a point $p \in J^{1}\left(R^{2}, R^{2}\right)$ satisfying $p_{1}^{1}(p) \neq 0$, (9.1) is written by

$$
\begin{aligned}
& \alpha_{1}(x)+\beta_{1}(x) z_{2}+\alpha_{2}(x) z_{3}+\beta_{2}(x) z_{4}=0, \\
& \gamma_{1}(x)+\delta_{1}(x) z_{2}+\gamma_{2}(x) z_{3}+\delta_{2}(x) z_{4}=0
\end{aligned}
$$

where $z_{i}=z_{i} / z_{1}(i=2,3,4)$ with $z_{1}=p_{1}^{1}, \quad z_{2}=p_{1}^{2}, \quad z_{3}=p_{2}^{1}$ and $z_{4}=p_{2}^{2}$. Remember that (9.2) is pseudoelliptic at $x_{0}$, pseudoinvolutive at any point $p \in I(P)$ and satisfies $\operatorname{det} K^{i}\left(x_{0}\right) \neq 0, i=1,2$.

TheOREM 9.1. Let $p$ be a point of $J^{1}\left(R^{2}, R^{2}\right)$ such that $\alpha^{1}(p)$ is near to $x_{0}, z_{1}(p) \neq 0$ and $\left(\left(z_{2}\right)^{2}+a_{z_{2}}-b\right)(p) \neq 0$. Then around $p$ the system of differential equations (9.2) is written by

$$
\begin{aligned}
& I_{1}^{a, b}=\left(\beta_{2}(x) \delta_{1}(x)-\beta_{1}(x) \delta_{2}(x)\right) \operatorname{det}\left(a_{2}(x)\right)^{-1}, \\
& I_{2}^{a, b}=\left(\beta_{1}(x) \gamma_{2}(x)-\alpha_{2}(x) \delta_{1}(x)\right) \operatorname{det}\left(a_{2}(x)\right)^{-1}
\end{aligned}
$$

where $\left\{x_{1}, x_{2}, I_{1}^{a, b}, I_{2}^{a, b}\right\}$ is a fundamental system of differential invariants of $\mathfrak{U}(P)$ around $p$ given in Proposition 8.1.

Proof. We can write

$$
\begin{aligned}
& I_{1}^{a, b}=A_{1{ }^{3} 3}+A_{2 \mho_{4}}, \\
& I_{2}^{a, b}=B_{1 \vec{\partial}_{3}}+B_{2 \vec{\partial}_{4}}
\end{aligned}
$$

where, by setting $\rho_{1}=\left(a+\sqrt{a^{2}+4 b}\right) / 2$ and $\rho_{2}=\left(a-\sqrt{a^{2}+4 b}\right) / 2$,

$$
\begin{aligned}
& A_{1}=z_{2}\left(\rho_{1}+z_{2}\right)^{-1}\left(\rho_{2}+z_{2}\right)^{-1}, \\
& A_{2}=-\left(\rho_{1}+z_{2}\right)^{-1}\left(\rho_{2}+z_{2}\right)^{-1}, \\
& B_{1}=\rho_{1} \rho_{2}\left(\rho_{1}+z_{2}\right)^{-1}\left(\rho_{2}+z_{2}\right)^{-1}, \\
& B_{2}=\left(\rho_{1}+\rho_{2}+z_{2}\right)\left(\rho_{1}+z_{2}\right)^{-1}\left(\rho_{2}+z_{2}\right)^{-1} .
\end{aligned}
$$

Note that among $A_{1}, A_{2}, B_{1}$ and $B_{2}$ there exist the relations

$$
\begin{aligned}
& B_{1}=-\rho_{1} \rho_{2} A_{2}, \\
& B_{2}=A_{1}-\left(\rho_{1}+\rho_{2}\right) A_{2} .
\end{aligned}
$$


From (9.3) and (9.4) we have

$$
\begin{aligned}
& \jmath_{3}=\left(-\left(A_{1}-\left(\rho_{1}+\rho_{2}\right) A_{2}\right) I_{1}^{a, b}+A_{2} I_{2}^{a, b}\right)\left(A_{1}-\rho_{1} A_{2}\right)^{-1}\left(A_{1}-\rho_{2} A_{2}\right)^{-1}, \\
& \jmath_{4}=\left(-\rho_{1} \rho_{2} A_{2} I_{1}^{a, b}-A_{1} I_{2}^{a, b}\right)\left(A_{1}-\rho_{1} A_{2}\right)^{-1}\left(A_{1}-\rho_{2} A_{2}\right)^{-1} .
\end{aligned}
$$

Then by (9.5), the system of differential equations (9.2) is written by

$$
\left\{\begin{array}{l}
\left(-\alpha_{2}(x)\left(A_{1}-\left(\rho_{1}+\rho_{2}\right) A_{2}\right)-\beta_{2}(x) \rho_{1} \rho_{2} A_{2}\right) I_{1}^{a, b}+\left(\alpha_{2}(x) A_{2}-\beta_{2}(x) A_{1}\right) I_{2}^{a, b} \\
\quad=-\left(\alpha_{1}(x)+\beta_{1}(x) z_{2}\right)\left(A_{1}-\rho_{1} A_{2}\right)\left(A_{1}-\rho_{2} A_{2}\right) \\
\left(-\gamma_{2}(x)\left(A_{1}-\left(\rho_{1}+\rho_{2}\right) A_{2}\right)-\delta_{2}(x) \rho_{1} \rho_{2} A_{2}\right) I_{1}^{a, b}+\left(\gamma_{2}(x) A_{2}-\delta_{2}(x) A_{1}\right) I_{2}^{a, b} \\
\quad=-\left(\gamma_{1}(x)+\delta_{1}(x) z_{2}\right)\left(A_{1}-\rho_{1} A_{2}\right)\left(A_{1}-\rho_{2} A_{2}\right) .
\end{array}\right.
$$

Now let us solve (9.6) with respect to $I_{1}^{a, b}$ and $I_{2}^{a, b}$. We have

$$
\begin{aligned}
& \left|\begin{array}{ll}
\alpha_{2}(x)\left(A_{1}-\left(\rho_{1}+\rho_{2}\right) A_{2}\right)+\beta_{2}(x) \rho_{1} \rho_{2} A_{2} & -\alpha_{2}(x) A_{2}+\beta_{2}(x) A_{1} \\
\gamma_{2}(x)\left(A_{1}-\left(\rho_{1}+\rho_{2}\right) A_{2}\right)+\delta_{2}(x) \rho_{1} \rho_{2} A_{2} & -\gamma_{2}(x) A_{2}+\delta_{2}(x) A_{1}
\end{array}\right| \\
& \quad=\left(\alpha_{2}(x) \delta_{2}(x)-\gamma_{2}(x) \beta_{2}(x)\right)\left(A_{1}-\rho_{1} A_{2}\right)\left(A_{1}-\rho_{2} A_{2}\right), \\
& \left|\begin{array}{ll}
-\left(\alpha_{1}(x)+\beta_{1}(x) z_{2}\right)\left(A_{1}-\rho_{1} A_{2}\right)\left(A_{1}-\rho_{2} A_{2}\right) & -\alpha_{2}(x) A_{2}+\beta_{2}(x) A_{1} \\
-\left(\gamma_{1}(x)+\delta_{1}(x) z_{2}\right)\left(A_{1}-\rho_{1} A_{2}\right)\left(A_{1}-\rho_{2} A_{2}\right) & -\gamma_{2}(x) A_{2}+\delta_{2}(x) A_{1}
\end{array}\right| \\
& \quad=\left(A_{1}-\rho_{1} A_{2}\right)\left(A_{1}-\rho_{2} A_{2}\right) W_{1}
\end{aligned}
$$

where

$$
\begin{aligned}
W_{1}= & \left(\gamma_{1}(x) \beta_{2}(x)-\alpha_{1}(x) \delta_{2}(x)\right) A_{1}+\left(\delta_{1}(x) \beta_{2}(x)-\beta_{1}(x) \delta_{2}(x)\right) A_{1} \jmath_{2} \\
& +\left(\alpha_{1}(x) \gamma_{2}(x)-\gamma_{1}(x) \alpha_{2}(x)\right) A_{2}+\left(\beta_{1}(x) \gamma_{2}(x)-\delta_{1}(x) \alpha_{2}(x)\right) A_{2 \gamma_{2}}
\end{aligned}
$$

and

$$
\left|\begin{array}{cc}
\alpha_{2}(x)\left(A_{1}-\left(\rho_{1}+\rho_{2}\right) A_{2}\right) & -\left(\alpha_{1}(x)+\beta_{1}(x) z_{2}\right) \\
+\beta_{2}(x) \rho_{1} \rho_{2} A_{2} & \times\left(A_{1}-\rho_{1} A_{2}\right)\left(A_{1}-\rho_{2} A_{2}\right) \\
\gamma_{2}(x)\left(A_{1}-\left(\rho_{1}+\rho_{2}\right) A_{2}\right) & -\left(\gamma_{1}(x)+\delta_{1}(x) z_{2}\right) \\
+\delta_{2}(x) \rho_{1} \rho_{2} A_{2} & \times\left(A_{1}-\rho_{1} A_{2}\right)\left(A_{1}-\rho_{2} A_{2}\right)
\end{array}\right|
$$

where

$$
\begin{aligned}
W_{2}= & \left(\alpha_{1}(x) \gamma_{2}(x)-\alpha_{2}(x) \gamma_{1}(x)\right) A_{1}+\left(\alpha_{1}(x)\left(\delta_{2}(x) \rho_{1} \rho_{2}-\gamma_{2}(x)\left(\rho_{1}+\rho_{2}\right)\right)\right. \\
& \left.-\gamma_{1}(x)\left(\beta_{2}(x) \rho_{1} \rho_{2}-\alpha_{2}(x)\left(\rho_{1}+\rho_{2}\right)\right)\right) A_{2} \\
& +\left(\beta_{1}(x) \gamma_{2}(x)-\alpha_{2}(x) \delta_{1}(x)\right) A_{1} \partial_{2}+\left(\beta_{1}(x)\left(\delta_{2}(x) \rho_{1} \rho_{2}-\gamma_{2}(x)\left(\rho_{1}+\rho_{2}\right)\right)\right. \\
& \left.-\delta_{1}(x)\left(\beta_{2}(x) \rho_{1} \rho_{2}-\alpha_{2}(x)\left(\rho_{1}+\rho_{2}\right)\right)\right) A_{2} \partial_{2} .
\end{aligned}
$$

Since we have 


$$
\left\{\begin{aligned}
\rho_{1} \rho_{2}=- & b \\
= & \left(\alpha_{1}(x) \gamma_{2}(x)-\alpha_{2}(x) \gamma_{1}(x)\right)\left(\delta_{2}(x) \beta_{1}(x)-\beta_{2}(x) \delta_{1}(x)\right)^{-1} \\
\rho_{1}+\rho_{2}= & a \\
= & \left(\gamma_{2}(x) \beta_{1}(x)-\alpha_{2}(x) \delta_{1}(x)+\alpha_{1}(x) \delta_{2}(x)-\beta_{2}(x) \gamma_{1}(x)\right) \\
& \times\left(\delta_{2}(x) \beta_{1}(x)-\beta_{2}(x) \delta_{1}(x)\right)^{-1}
\end{aligned}\right.
$$

we can write

$$
\begin{aligned}
A_{2} & =-\left(\rho_{1} \rho_{2}+\left(\rho_{1}+\rho_{2}\right) \jmath_{2}+\left(\jmath_{2}\right)^{2}\right)^{-1} \\
& =\left(\beta_{2}(x) \delta_{1}(x)-\delta_{2}(x) \beta_{1}(x)\right) U^{-1}
\end{aligned}
$$

where

$$
\begin{aligned}
U= & \alpha_{1}(x) \gamma_{2}(x)-\alpha_{2}(x) \gamma_{1}(x) \\
& +\left(\gamma_{2}(x) \beta_{1}(x)-\alpha_{2}(x) \delta_{1}(x)+\alpha_{1}(x) \delta_{2}(x)-\beta_{2}(x) \gamma_{1}(x)\right) z_{2} \\
& +\left(\delta_{2}(x) \beta_{1}(x)-\beta_{2}(x) \delta_{1}(x)\right)\left(z_{2}\right)^{2} .
\end{aligned}
$$

Therefore the relation $A_{1}=-A_{2 \jmath_{2}}$ and an elementary calculation show that

$$
\begin{aligned}
-W_{1}= & A_{2}\left\{\left(\alpha_{1}(x) \delta_{2}(x)-\gamma_{1}(x) \beta_{2}(x)\right) \partial_{2}+\left(\beta_{1}(x) \delta_{2}(x)-\delta_{1}(x) \beta_{2}(x)\right)\left(\partial_{2}\right)^{2}\right. \\
& \left.+\left(\alpha_{1}(x) \gamma_{2}(x)-\gamma_{1}(x) \alpha_{2}(x)\right)+\left(\beta_{1}(x) \gamma_{2}(x)-\delta_{1}(x) \alpha_{2}(x)\right) \vec{\partial}_{2}\right\} \\
= & \beta_{2}(x) \delta_{1}(x)-\delta_{2}(x) \beta_{1}(x) .
\end{aligned}
$$

As for $W_{2}$, we have

$$
\begin{aligned}
W_{2}= & \left(\alpha_{1}(x) \gamma_{2}(x)-\alpha_{2}(x) \gamma_{1}(x)\right) A_{1}+V_{1} A_{2} \\
& +\left(\beta_{1}(x) \gamma_{2}(x)-\alpha_{2}(x) \delta_{1}(x)\right) A_{1 \partial_{2}}+V_{2} A_{2 \partial_{2}}
\end{aligned}
$$

where

$$
V_{1}=-\alpha_{1}(x)\left(\delta_{2}(x) b+\gamma_{2}(x) a\right)+\gamma_{1}(x)\left(\beta_{2}(x) b+\alpha_{2}(x) a\right)
$$

and

$$
V_{2}=-\beta_{1}(x)\left(\delta_{2}(x) b+\gamma_{2}(x) a\right)+\delta_{1}(x)\left(\beta_{2}(x) b+\alpha_{2}(x) a\right)
$$

By (9.10) we can easily get

$$
\left\{\begin{aligned}
V_{1}= & \left(\alpha_{2}(x) \gamma_{1}(x)-\alpha_{1}(x) \gamma_{2}(x)\right)\left(\gamma_{2}(x) \beta_{1}(x)-\alpha_{2}(x) \delta_{1}(x)\right) \\
& \times\left(\delta_{2}(x) \beta_{1}(x)-\beta_{2}(x) \delta_{1}(x)\right)^{-1} \\
V_{2}= & \left\{\left(\alpha_{1}(x) \delta_{1}(x)-\beta_{1}(x) \gamma_{1}(x)\right)\left(\alpha_{2}(x) \delta_{2}(x)-\beta_{2}(x) \gamma_{2}(x)\right)\right. \\
& \left.-\left(\alpha_{2}(x) \delta_{1}(x)-\beta_{1}(x) \gamma_{2}(x)\right)^{2}\right\}\left(\delta_{2}(x) \beta_{1}(x)-\beta_{2}(x) \delta_{1}(x)\right)^{-1}
\end{aligned}\right.
$$

Then from (9.11) 


$$
\begin{aligned}
V_{1} A_{2}+V_{2} A_{2 \mho_{2}}= & \left(\alpha_{1}(x) \gamma_{2}(x)-\alpha_{2}(x) \gamma_{1}(x)\right)\left(\gamma_{2}(x) \beta_{1}(x)-\alpha_{2}(x) \delta_{1}(x)\right) U^{-1} \\
& +\left(\left(\alpha_{2}(x) \delta_{1}(x)-\beta_{1}(x) \gamma_{2}(x)\right)^{2}-\left(\alpha_{1}(x) \delta_{1}(x)-\beta_{1}(x) \gamma_{1}(x)\right)\right. \\
& \left.\times\left(\alpha_{2}(x) \delta_{2}(x)-\beta_{2}(x) \gamma_{2}(x)\right)\right){ }_{\gamma_{2}} U^{-1} .
\end{aligned}
$$

Therefore, by the relation $A_{1}=-A_{2 \mho_{2}}$ again, we get

$$
\begin{aligned}
-W_{2}= & U^{-1}\left\{\alpha_{1}(x) \gamma_{2}(x)-\alpha_{2}(x) \gamma_{1}(x)\right)\left(\delta_{2}(x) \beta_{1}(x)-\beta_{2}(x) \delta_{1}(x)\right)_{\partial_{2}} \\
& +\left(\beta_{1}(x) \gamma_{2}(x)-\alpha_{2}(x) \delta_{1}(x)\right)\left(\delta_{2}(x) \beta_{1}(x)-\beta_{2}(x) \delta_{1}(x)\right)\left(\mho_{2}\right)^{2} \\
& +\left(\alpha_{1}(x) \gamma_{2}(x)-\alpha_{2}(x) \gamma_{1}(x)\right)\left(\gamma_{2}(x) \beta_{1}(x)-\alpha_{2}(x) \delta_{1}(x)\right) \\
& +\left(\left(\alpha_{2}(x) \delta_{1}(x)-\beta_{1}(x) \gamma_{2}(x)\right)^{2}\right. \\
& \left.\left.\quad-\left(\alpha_{1}(x) \delta_{1}(x)-\beta_{1}(x) \gamma_{1}(x)\right)\left(\alpha_{2}(x) \delta_{2}(x)-\beta_{2}(x) \gamma_{2}(x)\right)\right) \partial_{2}\right\} \\
= & \gamma_{2}(x) \beta_{1}(x)-\alpha_{2}(x) \delta_{1}(x) .
\end{aligned}
$$

Then by (9.7), (9.8) and (9.9), we see that (9.6) is written by

$$
\begin{aligned}
& I_{1}^{a, b}=\left(\beta_{2}(x) \delta_{1}(x)-\beta_{1}(x) \delta_{2}(x)\right) \operatorname{det}\left(a_{2}(x)\right)^{-1}, \\
& I_{2}^{a, b}=\left(\beta_{1}(x) \gamma_{2}(x)-\alpha_{2}(x) \delta_{1}(x)\right) \operatorname{det}\left(a_{2}(x)\right)^{-1} .
\end{aligned}
$$

This completes the proof of Theorem 9.1.

\section{$\S 4$. Geometric structures of differential operators of infinite type}

10. In this section we shall investigate a geometric structure of a differential operator satisfying the condition (1), (2), (3) and (4) stated at the beginning of the article 6 .

Proposition 10.1. Let $P(x, D)$ be a differential operator of infinite type at $x_{0}$ with the structure vector $(a, b)$. Let $p$ be a point of $J^{1}\left(R^{2}, R^{2}\right)$ such that $\alpha^{1}(p)$ is near to $x_{0}, p_{1}^{1}(p) \neq 0$ and $\left(\left(\hat{\delta}_{2}\right)^{2}+a_{\partial_{2}}-b\right)(p) \neq 0$. Then a vector field $Z$ given around $\beta^{1}(p) \in R^{2}$ is a local cross-section of $\mathfrak{\Omega}_{\mathfrak{U}(P)}$ if and only if it satisfies the relations $Z^{(1)} I_{1}^{a, b}=0$ and $Z^{(1)} I_{2}^{a, b}=0$ around $p$.

Proof. By Theorem 9.1, the system of differential equations (9.2) is written by

$$
\begin{aligned}
& I_{1}^{a, b}=\left(\beta_{2}(x) \delta_{1}(x)-\beta_{1}(x) \delta_{2}(x)\right) \operatorname{det}\left(a_{2}(x)\right)^{-1}, \\
& I_{2}^{a, b}=\left(\beta_{1}(x) \gamma_{2}(x)-\alpha_{2}(x) \delta_{1}(x)\right) \operatorname{det}\left(a_{2}(x)\right)^{-1} .
\end{aligned}
$$

Then if we denote by $\Gamma$ the pseudogroup on a neighbourhood of $\beta^{1}(p) \in R^{2}$ defined by $\Gamma \ni \phi$ if and only if $\phi^{(1) *} I_{1}^{a, b}=I_{1}^{a, b}$ and $\phi^{(1) *} I_{2}^{a, b}=I_{2}^{a, b}$, it is clear that $\Gamma \subset \mathfrak{U}(P)$ around $\beta^{1}(p)$. Conversely by Proposition 8.1, $I_{1}^{a, b}$ and $I_{2}^{a, b}$ are differential invariants of $\mathfrak{U}(P)$ around $p$. Therefore $\mathfrak{L}_{\mathfrak{M}(P)} \subset \mathfrak{L}_{\Gamma}$ around $\beta^{1}(p)$. This proves that $\mathfrak{L}_{\mathfrak{r}(P)}=\mathfrak{L}_{\Gamma}$ around $\beta^{1}(p)$. 
11. At the beginning of this article we shall define automorphic systems which will play an important role hereafter.

Let $N$ and $M$ be $C^{\infty}$-manifolds with $\operatorname{dim} N=\operatorname{dim} M$ and denote by $\tilde{J}^{k}(N, M)$ the space of $k$-jets of local diffeomorphisms of $N$ to $M$.

Let $\Gamma$ be a pseudogroup on $M$. Then there corresponds to $\Gamma$ a weak Lie algebra sheaf $\mathfrak{L}_{\Gamma}$ on $M$. If we denote by $\mathfrak{P}\left(\mathfrak{L}_{\Gamma}\right)$ the pseudogroup on $M$ generated by local 1-parameter groups of local transformations which induce local cross-sections of $\mathfrak{L}_{\Gamma}$, then $\mathfrak{B}\left(\mathfrak{R}_{\Gamma}\right) \subset \Gamma$.

Let $\left\{F_{j}\right\}_{j=1}^{r}$ be a family of functions defined on a neighbourhood of $p \in \widetilde{J}^{k}(N, M)$.

Definition 11.1. A system of differential equations

$$
P: \quad F_{1}=0, \cdots, F_{r}=0
$$

is said to be $\Gamma$-automorphic at $\left(x_{0}, f\right)$ if the following conditions (i), (ii) and (iii) are satisfied:

(i) $x_{0} \in N$ and $f$ is a solution of $P$ around $x_{0}$.

(ii) Any solution of $P$ near to $f$ is written by $\phi \circ f$ for some $\phi \in \Gamma$ near to the identity.

(iii) For any $\psi \in \mathfrak{P}\left(\mathfrak{L}_{\Gamma}\right)$, if the composite $\psi \circ f$ is defined, it is a solution of $P$.

Remember that we denote by $\mathscr{S}(P)$ the solution space of $P$ and by $\mathfrak{U}(P)$ the pseudogroup generated by all local transformations $\phi: M \supset \mathfrak{H} \rightarrow$ $\mathfrak{B} \subset M$ satisfying $\phi(\mathfrak{S}(P)|\mathfrak{U}=\mathfrak{S}(P)| \mathfrak{B} . \quad \mathfrak{A}(P)$ is called the automorphism pseudogroup of $P$. Since $P$ is given on $\widetilde{J}^{k}(N, M)$, any solution of $P$ is a local diffeomorphism of $N$ to $M$.

Proposition 11.1. Assume that $\Gamma$ is ( $N, k)$-regular (cf. Definition 5.1) and let $\left\{x_{1}, \cdots, x_{n}, y_{1}, \cdots, y_{m}\right\}$ be a fundamental system of differential invariants of $\Gamma$ around $p=j_{x_{0}}^{k}(f) \in \tilde{J}^{k}(N, M)$ where $\left\{x_{1}, \cdots, x_{n}\right\}$ is a local coordinate system at $x_{0} \in N$. Consider the system of differential equations

$$
P: \quad y_{1}=\lambda_{1}, \cdots, y_{m}=\lambda_{m}
$$

where $\lambda_{j}(x)=y_{j}\left(j_{x}^{k}(f)\right), j=1, \cdots, m$. Around $f\left(x_{0}\right)$ if, near the identity transformation, $\Gamma$ is defined by the condition that $\Gamma \ni \phi$ if and only if $\phi^{(1)} * y_{j}=y_{j}, j=1, \cdots, m$, then $P$ is $\Gamma$-automorphic at $\left(x_{0}, f\right)$.

Proof. Let $f^{1}$ and $f^{2}$ be solutions of $P$ defined on a neighbourhood $U$ of $x_{\diamond}$. Then we have 


$$
y_{j}\left(j_{x}^{k}\left(f^{1}\right)\right)=y_{j}\left(j_{x}^{k}\left(f^{2}\right)\right), \quad j=1, \cdots, m
$$

for any $x \in U$. Let $U^{k}$ be a neighbourhood of $p$ such that $U^{k} / D_{\Gamma}^{(k)}$ (cf. Definition 5.1) is a manifold and let $\pi^{k}$ be the projection of $U^{k}$ onto $U^{k} / D_{\Gamma}^{(k)}$. Then (11.1) implies that $\pi^{k}\left(j_{x}^{k}\left(f^{1}\right)\right)=\pi^{k}\left(j_{x}^{k}\left(f^{2}\right)\right)$. Let $\omega$ be any local diffeomorphism of $U$ and define a local transformation $\omega^{k}$ of $J^{k}(N, M)$ by $\omega^{k}\left(j_{x}^{k}(f)\right)=j_{\omega^{-1}(x)}^{k}(f \circ \omega)$. Then for any local transformation $\phi$ of $M$, in particular for $\phi \in \Gamma$, we have $\phi^{(k)} \circ \omega^{k}=\omega^{k} \circ \phi^{(k)}$ if the composite is defined. This means that we have $\pi^{k}\left(j_{x}^{k}\left(f^{1} \circ \omega\right)\right)=\pi^{k}\left(j_{x}^{k}\left(f^{2} \circ \omega\right)\right)$ for any $x$ in the domain $D(\omega)$ of $\omega$. We set $g=f^{2} \circ\left(f^{1}\right)^{-1}$. Then we have

$$
\begin{aligned}
g^{(k)} j_{x}^{k}\left(f^{1} \circ \omega\right) & =j_{x}^{k}\left(f^{2} \circ\left(f^{1}\right)^{-1} \circ f^{1} \circ \omega\right) \\
& =j_{x}^{k}\left(f^{2} \circ \omega\right), \quad x \in D(\omega) .
\end{aligned}
$$

Therefore we get

$$
\pi^{k}\left(g^{(k)} j_{x}^{k}\left(f^{1} \circ \omega\right)\right)=\pi^{k}\left(j_{x}^{k}\left(f^{1} \circ \omega\right)\right), \quad x \in D(\omega)
$$

or

$$
\begin{aligned}
\left(g^{(k) * y_{j}^{k}}\left(j_{x}^{k}\left(f^{1} \circ \omega\right)\right)=y_{j}^{k}\left(j_{x}^{k}\left(f^{1} \circ \omega\right)\right), \quad\right. & x \in D(\omega), \\
& j=1, \cdots, m .
\end{aligned}
$$

Since $\omega$ is any local transformation on $U$, we have

$$
g^{(k) * y_{j}^{k}}=y_{j}^{k}, \quad j=1, \cdots, m .
$$

If $f^{1}$ and $f^{2}$ are near to $f$, then $g$ is near to the identity. Therefore (11.2) implies that $g$ belongs to $\Gamma$. This completes the proof.

12. Now again we assume that $N=M=R^{2}$. Let $(a, b) \in R^{2}$ and denote by $\mathfrak{P}_{(a, b)}$ the pseudogroup on $R^{2}$ defined by the condition that $\mathfrak{P}_{(a, b)} \ni \phi=\left(\phi_{1}\left(u_{1}, u_{2}\right), \phi_{2}\left(u_{1}, u_{2}\right)\right)$ if and only if $\partial \phi_{1} / \partial u_{1}=a\left(\partial \phi_{1} / \partial u_{2}\right)+\partial \phi_{2} / \partial u_{2}$ and $\partial \phi_{2} / \partial u_{1}=b\left(\partial \phi_{1} / \partial u_{2}\right)$.

Let us consider a differential operator $P(x, D)$ of $\Gamma\left(2, R^{2}\right)$ to $\Gamma\left(2, R^{2}\right)$ of order 1 satisfying the following conditions:

(i) $P(x, D)$ admits a solution $f$ which is a local diffeomorphism of $R^{2}$ to $R^{2}$ defined on a neighbourhood of $x_{0}$.

(ii) The automorphism pseudogroup $\mathfrak{X}(P)$ of $P(x, D)$ is $\mathfrak{P}_{(a, b)}$ for some vector $(a, b) \in R^{2}$ satisfying $\left(\left(z_{2}\right)^{2}+a_{z_{2}}-b\right)(p) \neq 0$ where $p=j_{x_{0}}^{1}(f)$.

THEOREM 12.1. If $P(x, D)$ above is of infinite type at $x_{0}$ with the structure vector $(a, b)$, the system of differential equations $P: P(x, D) u=0$ is 
$\mathfrak{U}(P)$-automorphic at $\left(x_{0}, f\right)$. Conversely, under the analyticity, for a differential operator $P(x, D)$ satisfying (i) and (ii), if $P: P(x, D) u=0$ is $\mathfrak{X}(P)$ automorphic at $\left(x_{0}, f\right)$ then there exists a differential operator $\tilde{P}(x, D)$ of infinite type at $x_{0}$ with the structure vector $(a, b)$ such that $\widetilde{S}(\tilde{P})=\widetilde{S}(P)$ near $f$.

Before proving Theorem 12.1, the following lemma is prepared.

Lemma 12.2. For any vector $(a, b) \in R^{2}$ there exists a differential operator $P(x, D)$ of infinite type at $x_{0}$ with the structure vector $(a, b)$.

Proof. Choose arbitrary constants $\beta_{1}, \beta_{2}, \delta_{1}$ and $\delta_{2}$ satisfying $\delta_{2} \beta_{1}-$ $\beta_{2} \delta_{1} \neq 0$ and consider the following equations with variables $X_{1}, X_{2}, X_{3}$ and $X_{4}$ :

$$
\begin{aligned}
& \delta_{2} X_{1}-\delta_{1} X_{2}-\beta_{2} X_{3}+\beta_{1} X_{4}=a\left(\delta_{2} \beta_{1}-\beta_{2} \delta_{1}\right), \\
& X_{2} X_{3}-X_{1} X_{4}=b\left(\delta_{2} \beta_{1}-\beta_{2} \delta_{1}\right) .
\end{aligned}
$$

It is easy to see that the set of points $\left(X_{1}, X_{2}, X_{3}, X_{4}\right) \in R^{4}$ satisfying (12.1) contains such a point $\left(X_{1}^{0}, X_{2}^{0}, X_{3}^{0}, X_{4}^{0}\right)$ that $\delta_{1} X_{1}^{0}-\beta_{1} X_{3}^{0} \neq 0, \delta_{2} X_{2}^{0}-\beta_{2} X_{4}^{0} \neq 0$, $\beta_{1} X_{2}^{0}-\beta_{2} X_{1}^{0} \neq 0$ and $\delta_{1} X_{4}^{0}-\delta_{2} X_{3}^{0} \neq 0$. Then if we set $\alpha_{1}=X_{1}^{0}, \alpha_{2}=X_{2}^{0}$, $\gamma_{1}=X_{3}^{0}$ and $\gamma_{2}=X_{4}^{0}$, the system of differential equations

$$
P_{0}: \begin{aligned}
& \alpha_{1} p_{1}^{1}+\beta_{1} p_{1}^{2}+\alpha_{2} p_{2}^{1}+\beta_{2} p_{2}^{2}=0, \\
& \gamma_{1} p_{1}^{1}+\delta_{1} p_{1}^{2}+\gamma_{2} p_{2}^{1}+\delta_{2} p_{2}^{2}=0
\end{aligned}
$$

is pseudoelliptic at any $x \in R^{2}$, pseudoinvolutive at any $p \in I\left(P_{0}\right)$ and satisfies $\operatorname{det} K^{i}(x) \neq 0$. Then by Theorem 4.1 and Remark $6.1, P_{0}$ is of infinite type at any $x_{0} \in R^{2}$ with the structure vector $(a, b)$. This completes the proof of Lemma 12.2 .

13. Proof of Theorem 12.1. Assume that $P(x, D)$ is of infinite type at $x_{0}$ with the structure vector $(a, b)$. By Theorem 9.1 the system of differential equations $P$ i.e. (9.2) is written by

$$
\begin{aligned}
& I_{1}^{a, b}=\left(\beta_{2}(x) \delta_{1}(x)-\beta_{1}(x) \delta_{2}(x)\right) \operatorname{det}\left(a_{2}(x)\right)^{-1}, \\
& I_{2}^{a, b}=\left(\beta_{1}(x) \gamma_{2}(x)-\alpha_{2}(x) \delta_{1}(x)\right) \operatorname{det}\left(a_{2}(x)\right)^{-1}
\end{aligned}
$$

where $\left\{x_{1}, x_{2}, I_{1}^{a, b}, I_{2}^{a, b}\right\}$ is a fundamental system of differential invariants of $\mathfrak{X}(P)$ around $p$. Then by Proposition 10.1 and Proposition 11.1, we see that $P$ is $\mathfrak{U}(P)$-automorphic at $\left(x_{0}, f\right)$.

Conversely we assume that the system of differential equations $P$ is $\mathfrak{Y}(P)$-automorphic at $\left(x_{0}, f\right)$. By Lemma 12.2 there exists a differential 
operator $\bar{P}(x, D)$ of infinite type at $x_{0}$ with structure vector $(a, b)$. Then by Proposition 10.1, the pseudogroup $\mathfrak{P}_{(a, b)}$ is, near the identity, defined as the set of local transformations $\phi$ on $R^{2}$ satisfying $\phi^{(1) *} I_{i}^{b, b}=I_{i}^{b, b}, i=$ 1,2. Therefore by Proposition 11.1, the system of differential equations

$$
I_{1}^{a, b}=\lambda_{1}, \quad I_{2}^{a, b}=\lambda_{2}
$$

where $\lambda_{i}(x)=I_{i}^{a, b}\left(j_{x}^{1}(f)\right), i=1,2$, is $\mathfrak{R}(P)$-automorphic at $\left(x_{0}, f\right)$. Since $f$ is a solution of $P: P(x, D) u=0$, the system of differential equations $P$ is written by (13.1) around $p$ and near $f$. Note that $\lambda_{1}(x) \neq 0$ because $f$ is a diffeomorphism and so satisfies $z_{2} z_{3}-z_{4} \neq 0$ at $j_{x}^{1}(f)$.

Let $\tilde{a}_{2}^{21}$ and $\tilde{a}_{2}^{22}$ be any two constants satisfying $\tilde{a}_{2}^{21} \lambda_{1}\left(x_{0}\right)+\tilde{a}_{2}^{22} \lambda_{2}\left(x_{0}\right) \neq 0$. We set

$$
\begin{aligned}
& A(X, Y, x)=X \lambda_{1}(x)+Y \lambda_{2}(x), \\
& B(x)=\tilde{a}_{2}^{21} \lambda_{1}(x)+\tilde{a}_{2}^{22} \lambda_{2}(x)
\end{aligned}
$$

and

$$
\begin{aligned}
C(X, Y, x)= & \left(\tilde{a}_{2}^{22} X-\tilde{a}_{2}^{21} Y\right)^{-1}\left\{(a X+b Y)\left(A(X, Y, x) \tilde{a}_{2}^{22}-B(x) Y\right)\right. \\
& \left.+X\left(B(x) X-A(X, Y, x) \tilde{a}_{2}^{21}\right)\right\} \\
D(X, Y, x)= & \left(\tilde{a}_{2}^{22} X-\tilde{a}_{2}^{21} Y\right)^{-1}\left\{\left(b \tilde{a}_{2}^{22}+a \tilde{a}_{2}^{21}\right)\left(A(X, Y, x) \tilde{a}_{2}^{22}-B(x) Y\right)\right. \\
& \left.+\tilde{a}_{2}^{21}\left(B(x) X-A(X, Y, x) \tilde{a}_{2}^{21}\right)\right\} .
\end{aligned}
$$

Then it is easy to see that there exist constants $X_{0}$ and $Y_{0}$ satisfying the following conditions (13.4) (13.8):

$$
\begin{aligned}
& \tilde{a}_{2}^{22} X_{0}-\tilde{a}_{2}^{21} Y_{0} \neq 0 . \\
& B\left(x_{0}\right) C\left(X_{0}, Y_{0}, x_{0}\right)-A\left(X_{0}, Y_{0}, x_{0}\right) D\left(X_{0}, Y_{0}, x_{0}\right) \neq 0 . \\
& B\left(x_{0}\right) \tilde{a}_{2}^{21}-\tilde{a}_{2}^{22} D\left(X_{0}, Y_{0}, x_{0}\right) \neq 0 . \\
& A\left(X_{0}, Y_{0}, x_{0}\right) X_{0}-C\left(X_{0}, Y_{0}, x_{0}\right) Y_{0} \neq 0 . \\
& A\left(X_{0}, Y_{0}, x_{0}\right) \tilde{a}_{2}^{22}-B\left(x_{0}\right) Y_{0} \neq 0 .
\end{aligned}
$$

If we set $\tilde{a}_{1}^{12}(x)=A\left(X_{0}, Y_{0}, x\right), \quad \tilde{a}_{1}^{22}(x)=B(x), \quad \tilde{a}_{2}^{11}=X_{0}$ and $\tilde{a}_{2}^{12}=Y_{0}$, then (13.3) is written by

$$
\left\{\begin{array}{l}
C\left(X_{0}, Y_{0}, x\right)=-\operatorname{det}\left(\tilde{a}_{2}\right)^{-1}\left|\begin{array}{cc}
a\left(\tilde{a}_{1}^{12}(x) \tilde{a}_{2}^{22}-\tilde{a}_{1}^{22}(x) \tilde{a}_{2}^{12}\right) & -\tilde{a}_{2}^{12} \\
+\tilde{a}_{1}^{22}(x) \tilde{a}_{2}^{11}-\tilde{a}_{1}^{12}(x) \tilde{a}_{2}^{21} & \\
-b\left(\tilde{a}_{1}^{12}(x) \tilde{a}_{2}^{22}-\tilde{a}_{1}^{22}(x) \tilde{a}_{2}^{12}\right) & -\tilde{a}_{2}^{11}
\end{array}\right| \\
D\left(X_{0}, Y_{0}, x\right)=-\operatorname{det}\left(\tilde{a}_{2}\right)^{-1}\left|\begin{array}{cc}
\tilde{a}_{2}^{22} & a\left(\tilde{a}_{1}^{12}(x) \tilde{a}_{2}^{22}-\tilde{a}_{1}^{22}(x) \tilde{a}_{2}^{12}\right) \\
& +\tilde{a}_{1}^{22}(x) \tilde{a}_{2}^{11}-\tilde{a}_{1}^{12}(x) \tilde{a}_{2}^{21} \\
\tilde{a}_{2}^{21} & -b\left(\tilde{a}_{1}^{12}(x) \tilde{a}_{2}^{22}-\tilde{a}_{1}^{22}(x) \tilde{a}_{2}^{12}\right)
\end{array}\right|
\end{array}\right.
$$


By (13.2), $A\left(X_{0}, Y_{0}, x\right) \equiv \tilde{a}_{1}^{12}(x)$ and $B(x) \equiv \tilde{a}_{1}^{22}(x)$ satisfy

$$
\begin{aligned}
& \tilde{a}_{2}^{22} A\left(X_{0}, Y_{0}, x\right)-\tilde{a}_{2}^{12} B(x)=\lambda_{1}(x) \operatorname{det}\left(\tilde{a}_{2}\right) \\
& -\tilde{a}_{2}^{21} A\left(X_{0}, Y_{0}, x\right)+\tilde{a}_{2}^{11} B(x)=\lambda_{2}(x) \operatorname{det}\left(\tilde{a}_{2}\right)
\end{aligned}
$$

Therefore we get

$$
\begin{aligned}
& \left(\tilde{a}_{1}^{12}(x) \tilde{a}_{2}^{22}-\tilde{a}_{2}^{12} \tilde{a}_{1}^{22}(x)\right) \operatorname{det}\left(\tilde{a}_{2}\right)^{-1}=\lambda_{1}(x), \\
& \left(\tilde{a}_{2}^{11} \tilde{a}_{1}^{22}(x)-\tilde{a}_{1}^{12}(x) \tilde{a}_{2}^{21}\right) \operatorname{det}\left(\tilde{a}_{2}\right)^{-1}=\lambda_{2}(x)
\end{aligned}
$$

around $x_{0}$.

If we set $\tilde{a}_{1}^{11}(x)=C\left(X_{0}, Y_{0}, x\right)$ and $\tilde{a}_{1}^{21}(x)=D\left(X_{0}, Y_{0}, x\right)$, then by (13.6), (13.7) and (13.8), we get

$$
\begin{aligned}
\operatorname{det} K^{i}(x)= & \left(\tilde{a}_{1}^{11}(x) \tilde{a}_{1}^{22}(x)-\tilde{a}_{1}^{12}(x) \tilde{a}_{1}^{21}(x)\right)^{-1}\left(\tilde{a}_{2}^{i 1} \tilde{a}_{1}^{i 2}(x)-\tilde{a}_{2}^{i 2} \tilde{a}_{1}^{i 1}(x)\right) \\
& \times\left(\tilde{a}_{2}^{22} \tilde{a}_{1}^{12}(x)-\tilde{a}_{2}^{12} \tilde{a}_{1}^{22}(x)\right) \\
& \neq 0 \text { around } x_{0} .
\end{aligned}
$$

Now consider the system of differential equations

$$
\tilde{P}: \quad \begin{aligned}
& \tilde{a}_{1}^{11}(x) p_{1}^{1}+\tilde{a}_{1}^{12}(x) p_{1}^{2}+\tilde{a}_{2}^{11} p_{2}^{1}+\tilde{a}_{2}^{12} p_{2}^{2}=0, \\
& \tilde{a}_{1}^{21}(x) p_{1}^{1}+\tilde{a}_{1}^{22}(x) p_{1}^{2}+\tilde{a}_{2}^{21} p_{2}^{1}+\tilde{a}_{2}^{22} p_{2}^{2}=0 .
\end{aligned}
$$

Then $\tilde{P}$ is pseudoelliptic at $x_{0}$ and $\operatorname{det} K^{i}\left(x_{0}\right) \neq 0$. From (13.9), we obtain the relations

$$
\begin{aligned}
& \tilde{a}_{2}^{22} \tilde{a}_{1}^{11}(x)-\tilde{a}_{2}^{12} \tilde{a}_{1}^{21}(x)=a\left(\tilde{a}_{2}^{22} \tilde{a}_{1}^{12}(x)-\tilde{a}_{2}^{12} \tilde{a}_{1}^{22}(x)\right)+\tilde{a}_{2}^{11} \tilde{a}_{1}^{22}(x)-\tilde{a}_{2}^{21} \tilde{a}_{1}^{12}(x), \\
& \tilde{a}_{2}^{21} \tilde{a}_{1}^{11}(x)-\tilde{a}_{2}^{11} \tilde{a}_{1}^{21}(x)=b\left(\tilde{a}_{2}^{12} \tilde{a}_{1}^{22}(x)-\tilde{a}_{2}^{22} \tilde{a}_{1}^{12}(x)\right) .
\end{aligned}
$$

Furthermore under the analyticity $\tilde{P}$ is pseudoinvolutive at any point $p \in I(\tilde{P})$. Therefore by Theorem $9.1, \tilde{P}$ is written by

$$
\begin{aligned}
& I_{1}^{a, b}=\left(\tilde{a}_{1}^{12}(x) \tilde{a}_{2}^{22}-\tilde{a}_{2}^{12} \tilde{a}_{1}^{22}(x)\right) \operatorname{det}\left(\tilde{a}_{2}\right)^{-1}, \\
& I_{2}^{a, b}=\left(\tilde{a}_{2}^{11} \tilde{a}_{1}^{22}(x)-\tilde{a}_{1}^{12}(x) \tilde{a}_{2}^{21}\right) \operatorname{det}\left(\tilde{a}_{2}\right)^{-1} .
\end{aligned}
$$

This shows that the solution space $\widetilde{S}(\tilde{P})$ of the system of differential equations $\tilde{P}$ coincides with the solution space $\widetilde{S}(P)$ of the system of differential equations $P$ around $x_{0}$ and near $f$. This completes the proof of Theorem 12.1.

\section{§5. Differential equations whose invariant structures are families of pseudoelliptic systems}

14. Let $f$ be a local transformation on $R^{2}$ defined on a neighbourhood of $x_{0}$ and set $p=j_{x_{0}}^{1}(f)$. For a vector $(a, b)$ and the point $p$, we 
assume that $\left(\left(\partial_{2}\right)^{2}+a_{\partial_{2}}-b\right)(p) \neq 0$.

Let $F\left(u_{1}, u_{2}\right)$ be any function on $R^{2}$ such that $\left(\partial F / \partial u_{1}\right)^{2}+\left(\partial F / \partial u_{2}\right)^{2} \neq 0$ on any domain of $R^{2}$ and set

$$
\lambda(x)=F\left(I_{1}^{a, b}\left(j_{x}^{1}(f)\right), I_{2}^{a, b}\left(j_{x}^{1}(f)\right)\right)
$$

around $x_{0}$. Consider the single differential equation

$$
P(F): \quad F\left(I_{1}^{a, b}, I_{2}^{a, b}\right)=\lambda
$$

and the pseudogroup

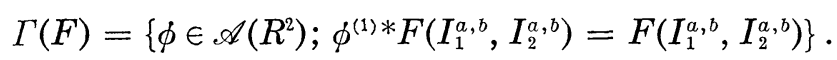

LEMMA 14.1. $\mathfrak{U}(P(F))=\Gamma(F)$.

Proof. It is clear that $\mathfrak{H}(P(F)) \supset \Gamma(F)$. Now let $\phi \in \mathfrak{U}(P(F))$. Then

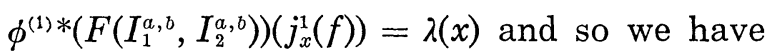

$$
\phi^{(1) *}\left(F\left(I_{1}^{a, b}, I_{2}^{a, b}\right)\right)\left(j_{x}^{1}(f)\right)=F\left(I_{1}^{a, b}, I_{2}^{a, b}\right)\left(j_{x}^{1}(f)\right) .
$$

Let $\omega$ be any local transformation on a neighbourhood $U$ of $x_{0}$. Then the local transformation $\omega^{1}$ on $J^{1}\left(R^{2}, R^{2}\right)$ defined by $\omega^{1}\left(j_{x}^{1}(s)\right)=j_{\omega-1(x)}^{1}(s \circ \omega)$ satisfies $\omega^{1} \circ \phi^{(1)}=\phi^{(1)} \circ \omega^{1}$. Therefore by (14.1) we get

$$
\begin{aligned}
& \phi^{(1) *}\left(F\left(I_{1}^{a, b}, I_{2}^{a, b}\right)\right)\left(j_{x}^{1}(f \circ \omega)\right)
\end{aligned}
$$

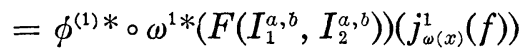

$$
\begin{aligned}
& =\omega^{1 *} \circ \phi^{(1) *}\left(F\left(I_{1}^{a, b}, I_{2}^{a, b}\right)\right)\left(j_{\omega(x)}^{1}(f)\right) \\
& =\omega^{1 *} F\left(I_{1}^{a, b}, I_{2}^{a, b}\right)\left(j_{\omega(x)}^{1}(f)\right) \\
& =F\left(I_{1}^{a, b}, I_{2}^{a, b}\right)\left(j_{x}^{1}(f \circ \omega)\right)
\end{aligned}
$$

where $x$ is any point in the domain of $\omega$. Since $\omega$ is an arbitrary local transformation on $U$, we have

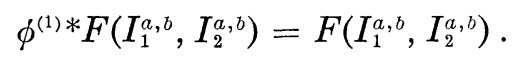

This shows that $\phi \in \Gamma(F)$ and completes the proof of Lemma 14.1.

15. Let $I$ and $J$ be differential invariants of $\mathfrak{P}_{(a, b)}$ defined on a neighbourhood of $j_{x_{0}}^{1}(f) \in J\left(R^{2}, R^{2}\right)$ such that $I+J \neq$ constant and $\partial I / \partial x_{i}=$ $\partial J / \partial x_{i}=0, i=1,2$ where $\left\{x_{1}, x_{2}, u_{1}, u_{2}, p_{1}^{1}, p_{1}^{2}, p_{2}^{1}, p_{2}^{2}\right\}$ is the canonical coordinate system on $J^{1}\left(R^{2}, R^{2}\right)$. Consider any vector field

$$
\begin{aligned}
Z= & \left(X_{u_{1}} p_{1}^{1}+X_{u_{2}} p_{1}^{2}\right)\left(\partial / \partial p_{1}^{1}\right)+\left(Y_{u_{1}} p_{1}^{1}+Y_{u_{2}} p_{1}^{2}\right)\left(\partial / \partial p_{1}^{2}\right) \\
& +\left(X_{u_{1}} p_{2}^{1}+X_{u_{2}} p_{2}^{2}\right)\left(\partial / \partial p_{2}^{1}\right)+\left(Y_{u_{1}} p_{2}^{1}+Y_{u_{2}} p_{2}^{2}\right)\left(\partial / \partial p_{2}^{2}\right)
\end{aligned}
$$


on $J^{1}\left(R^{2}, R^{2}\right)$. Then by setting $W_{1}=p_{1}^{1}\left(\partial / \partial p_{1}^{1}\right)+p_{2}^{1}\left(\partial / \partial p_{2}^{1}\right), W_{2}=p_{1}^{2}\left(\partial / \partial p_{1}^{1}\right)+$ $p_{2}^{2}\left(\partial / \partial p_{2}^{1}\right), W_{3}=p_{1}^{1}\left(\partial / \partial p_{1}^{2}\right)+p_{2}^{1}\left(\partial / \partial p_{2}^{2}\right)$ and $W_{4}=p_{1}^{2}\left(\partial / \partial p_{1}^{2}\right)+p_{2}^{2}\left(\partial / \partial p_{2}^{2}\right), Z$ is written by

$$
Z=X_{u_{1}} W_{1}+X_{u_{2}} W_{2}+Y_{u_{1}} W_{3}+Y_{u_{2}} W_{4} .
$$

Proposition 15.1. Assume that $b \neq 0$ and the following two conditions:

(i) $Z$ is a cross-section of $\mathfrak{Q}_{\mathfrak{B}_{(a, b)}}$ if and only if $Z I=0$.

(ii) $Z$ is a cross-section of $\mathcal{R}_{\mathfrak{P}_{(a, b)}}$ if and only if $Z J=0$.

Then $Z$ is a cross-section of $\mathfrak{Q}_{\mathfrak{P}_{(a, b)}}$ if and only if $Z(I+J)=0$.

Proof. By the condition (i), $Z I=0$ if and only if $X_{u_{1}}=a X_{u_{2}}+Y_{u_{2}}$ and $Y_{u_{1}}=b X_{u_{2}}$. On the other hand $Z I=0$ if and only if $X_{u_{1}} d I\left(W_{1}\right)+$ $X_{u_{2}} d I\left(W_{2}\right)+Y_{u_{1}} d I\left(W_{3}\right)+Y_{u_{2}} d I\left(W_{4}\right)=0$. Therefore, under the condition (i), we have

$$
\left(a X_{u_{2}}+Y_{u_{2}}\right) d I\left(W_{1}\right)+X_{u_{2}} d I\left(W_{2}\right)+b X_{u_{2}} d I\left(W_{3}\right)+Y_{u_{2}} d I\left(W_{4}\right)=0 .
$$

Since $X_{u_{2}}$ and $Y_{u_{2}}$ are arbitrary, we see that, under the condition (i), the following relations (15.1) hold:

$$
\begin{aligned}
& a d I\left(W_{1}\right)+d I\left(W_{2}\right)+b d I\left(W_{3}\right)=0, \\
& d I\left(W_{1}\right)+d I\left(W_{4}\right)=0 .
\end{aligned}
$$

Similarly, under the condition (ii), the following relations hold:

$$
\begin{aligned}
& \operatorname{adJ}\left(W_{1}\right)+d J\left(W_{2}\right)+b d J\left(W_{3}\right)=0, \\
& d J\left(W_{1}\right)+d J\left(W_{4}\right)=0 .
\end{aligned}
$$

Then we get the relations

$$
\begin{aligned}
& a d(I+J)\left(W_{1}\right)+d(I+J)\left(W_{2}\right)+b d(I+J)\left(W_{3}\right)=0, \\
& d(I+J)\left(W_{1}\right)+d(I+J)\left(W_{4}\right)=0 .
\end{aligned}
$$

Now we have

$$
\begin{aligned}
Z(I+J)= & X_{u_{1}} d(I+J)\left(W_{1}\right)+X_{u_{2}} d(I+J)\left(W_{2}\right) \\
& +Y_{u_{1}} d(I+J)\left(W_{3}\right)+Y_{u_{2}} d(I+J)\left(W_{4}\right) .
\end{aligned}
$$

Therefore by (15.3), we get

$$
\begin{aligned}
Z(I+J)= & \left(X_{u_{1}}-a X_{u_{2}}-Y_{u_{2}}\right) d(I+J)\left(W_{1}\right) \\
& +\left(Y_{u_{1}}-b X_{u_{2}}\right) d(I+J)\left(W_{3}\right) .
\end{aligned}
$$

We shall now prove that $d(I+J)\left(W_{1}\right) \neq 0$ and $d(I+J)\left(W_{3}\right) \neq 0$.

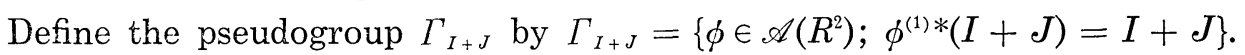


If $d(I+J)\left(W_{1}\right)=0$, then $W_{1}$ is a cross-section of $\mathfrak{R}_{\Gamma_{I+J}}$. On the other hand $\mathfrak{R}_{\Gamma_{I+J}} \supset \mathfrak{Q}_{\mathfrak{B}_{(a, b)}}$ and the vector fields $Z_{1}=p_{1}^{1}\left(\partial / \partial p_{1}^{1}\right)+p_{1}^{2}\left(\partial / \partial p_{1}^{2}\right)+p_{2}^{1}\left(\partial / \partial p_{2}^{1}\right)$ $+p_{2}^{2}\left(\partial / \partial p_{2}^{2}\right)$ and $Z_{2}=\left(a p_{1}^{1}+p_{1}^{2}\right)\left(\partial / \partial p_{1}^{1}\right)+b p_{1}^{1}\left(\partial / \partial p_{1}^{2}\right)+\left(a p_{2}^{1}+p_{2}^{2}\right)\left(\partial / \partial p_{2}^{1}\right)+b p_{2}^{1}\left(\partial / \partial p_{2}^{2}\right)$ are cross-sections of $\mathfrak{Q}_{\mathfrak{p}_{(a, b)}}$. Therefore $W_{1}, Z_{1}$ and $Z_{2}$ are cross-sections of $\mathfrak{R}_{\Gamma_{I+J}}$. Consider the space $\left(D_{\Gamma_{I+J}}^{(1)}\right)_{p}=\left(\mathfrak{R}_{\Gamma_{I+J}}^{(1)}\right)_{p} /\left(\mathbb{R}_{\Gamma_{I+J}}^{(1)}\right)_{p}^{0} \subset T_{p}\left(\tilde{J}^{1}\left(R^{2}, R^{2}\right)\right)$. Then $\left(D_{\Gamma_{I+J}}^{(1)}\right)_{p}$ is contained in the space of integral points $\mathfrak{\Im}(I+J-\lambda)$ of the differential equation $I+J=\lambda$ if $p \in \mathfrak{\Im}(I+J-\lambda)$ where $\lambda(x)=(I+J)\left(j_{x}^{1}(f)\right)$. Since the image of the map $j^{1}(s): D_{s}(=$ the domain of $s) \ni x \rightarrow j_{x}^{1}(s) \in$ $\tilde{J}^{1}\left(R^{2}, R^{2}\right)$ for any solution $s$ with rank 2 of the differential equation $I+$ $J=\lambda$ is transversal to $D_{\Gamma_{I+J}}^{(1)}, \operatorname{dim} D_{\Gamma_{I+J}}^{(1)}+2 \leq \operatorname{dim} \mathfrak{\Im}(I+J-\lambda)=7$ and so $\operatorname{dim} D_{T_{I+J}}^{(1)} \leq 5$.

Now $\partial / \partial u_{1}, \partial / \partial u_{2}, Z_{1}, Z_{2}$ and $W_{1}$ are cross-sections of $D_{\Gamma_{I+J}}^{(1)}$. There exists such a point $p \in \tilde{J}^{1}\left(R^{2}, R^{2}\right)$ that the tangent vectors $\left(\partial / \partial u_{1}\right)_{p}$, $\left(\partial / \partial u_{2}\right)_{p}$, $\left(Z_{1}\right)_{p},\left(Z_{2}\right)_{p}$ and $\left(W_{1}\right)_{p}$ are linearly independent and so, around $p, D_{\Gamma_{I+J}}^{(1)}$ is generated by $\partial / \partial u_{1}, \partial / \partial u_{2}, Z_{1}, Z_{2}, W_{1}$. Therefore $\left[W_{1} Z_{2}\right]$ is also a local crosssection of $D_{\Gamma_{I+J}}^{(1)}$ around $p$.

However we have $\left[W_{1}, Z_{2}\right]=b p_{1}^{1}\left(\partial / \partial p_{1}^{2}\right)+b p_{2}^{1}\left(\partial / \partial p_{2}^{2}\right)-p_{1}^{2}\left(\partial / \partial p_{1}^{1}\right)-p_{2}^{2}\left(\partial / \partial p_{2}^{1}\right)$ and this is not obtained by any linear combination of the above generator. This shows that $\left[W_{1}, Z_{2}\right]$ is not a local cross-section of $D_{I_{I+J}}^{(1)}$ around $p$ and this contradicts to the involutiveness of $D_{I_{I+J}}^{(1)}$. Therefore $d(I+J)\left(W_{1}\right) \neq 0$.

Similarly if $d(I+J)\left(W_{3}\right)=0$, around such a point $p$ that $\left(\partial / \partial u_{1}\right)_{p}$, $\left(\partial / \partial u_{2}\right)_{p},\left(Z_{1}\right)_{p},\left(Z_{2}\right)_{p}$ and $\left(W_{3}\right)_{p}$ are linearly independent, $\left\{\partial / \partial u_{1}, \partial / \partial u_{2}, Z_{1}, Z_{2}, W_{3}\right\}$ is a generator of $D_{\Gamma_{I+j}}^{(1)}$. However we have $\left[W_{3}, Z_{2}\right]=p_{1}^{1}\left(\partial / \partial p_{1}^{1}\right)+p_{2}^{1}\left(\partial / \partial p_{2}^{1}\right)-$ $\left(a p_{1}^{1}+p_{1}^{2}\right)\left(\partial / \partial p_{1}^{2}\right)-\left(a p_{2}^{1}+p_{2}^{2}\right)\left(\partial / \partial p_{2}^{2}\right)$ which is not obtained by any linear combination of the above generator. This contradicts to the involutiveness of $D_{\Gamma_{I+J}}^{(1)}$. Thus we get $d(I+J)\left(W_{3}\right) \neq 0$.

Now it is easy to see that $W_{1}(I+J)$ and $W_{3}(I+J)$ are independent as functions. Therefore by (15.4), $Z(I+J)=0$ if and only if $X_{u_{1}}=a X_{u_{2}}+$ $Y_{u_{2}}$ and $Y_{u_{1}}=b X_{u_{2}}$. This completes the proof of Proposition 15.1.

Corollary 15.2. Assume that $b \neq 0$ and $I^{m} J^{n} \neq$ constant where $m$ and $n$ are integers such that $m^{2}+n^{2} \neq 0$. Then under the conditions (i) and (ii) in Proposition 15.1, $Z\left(I^{m} J^{n}\right)=0$ if and only if $Z$ is a cross-section of $\mathfrak{Q}_{\mathfrak{w}_{(a, b)}}$.

Proof. Assume that $n=0$. Since we have $Z\left(I^{m}\right)=m I^{m-1} Z I, Z\left(I^{m}\right)=0$ if and only if $Z I=0$. Therefore by the condition (i), $Z\left(I^{m}\right)=0$ if and only if $Z$ is a cross-section of $\mathfrak{L}_{\mathfrak{B}_{(a, b)}}$. The similar assertion holds for the case $m=0$. 
Now assume that $m \neq 0$ and $n \neq 0$. Since $Z(\log |I|)=Z I / I, Z I=0$ if and only if $Z(\log |I|)=0$. Therefore $Z(m \log |I|)=0$ if and only if $Z$ is a cross-section of $\mathfrak{Q}_{\mathfrak{B}_{(a, b)}}$. Similarly $Z(n \log |J|)=0$ if and only if $Z$ is

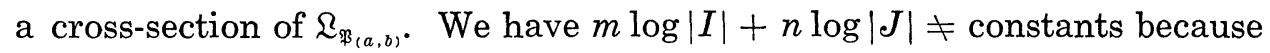
$I^{m} J^{n} \neq$ constant. Then by Proposition $15.1, Z(m \log |I|+n \log |J|)=0$ if and only if $Z$ is a cross-section of $\mathfrak{R}_{\mathfrak{P}_{(a, b)}}$.

On the other hand we have $Z(m \log |I|+n \log |J|)=Z\left(\log \left|I^{m} J^{n}\right|\right)=$ $Z\left(I^{m} J^{n}\right) / I^{m} J^{n}$. Therefore $Z\left(I^{m} J^{n}\right)=0$ if and only if $Z(m \log |I|+$ $n \log |J|)=0$. This completes the proof of Corollary 15.2.

CoROLlaRy 15.3. Let $F\left(z_{1}, z_{2}\right)$ be a non-constant real rational expression of the variables $z_{1}$ and $z_{2}$ i.e. $F\left(z_{1}, z_{2}\right)=\left(\sum_{i, j=1}^{s} a_{i j} z_{1}^{m_{i}} z_{2}^{m_{j}}\right) /\left(\sum_{k, \ell=1}^{t} b_{k \ell} z_{1}^{n_{k}} z_{2}^{n_{\ell}}\right)$ where $a_{i j}$ and $b_{k \ell}$ are non-zero real numbers and $m_{i}, n_{k}$ are integers $\geq 0$. Assume that $I$ and $J$ are functionally independent and $b \neq 0$. Then, under the conditions (i) and (ii) in Proposition 15.1, $Z(F(I, J))=0$ if and only if $Z$ is a cross-section of $\mathfrak{Q}_{\mathfrak{P}_{(a, b)}}$.

Proof. Since $I$ and $J$ are functionally independent, $\sum_{i, j=1}^{s^{\prime}} a_{i j} I^{m_{i}} J^{m_{j}}$ $\left(1 \leq s^{\prime} \leq s\right), \sum_{k, \ell=1}^{t^{\prime}} b_{k \ell} I^{n_{k}} J^{n_{\ell}}\left(1 \leq t^{\prime} \leq t\right)$ are not constants. Therefore by Proposition 15.1 and Corollary 15.2, $Z\left(\sum_{i, j=1}^{s} a_{i j} I^{m_{i}} J^{m_{j}}\right)=0$ if and only if $Z$ is a cross-section of $\mathfrak{\Re}_{\mathfrak{P}_{(a, b)}}$ and $Z\left(\sum_{k, \ell=1}^{t} b_{k \ell} I^{n_{k}} \boldsymbol{J}^{n_{\ell}}\right)=0$ if and only if $Z$ is a cross-section of $\mathfrak{L}_{\mathfrak{P}_{(a, b)}}$. Since $F(I, J)$ is not constant, again by Corollary $15.2, Z(F(I, J))=0$ if and only if $Z$ is a cross-section of $\mathfrak{Q}_{\mathfrak{P}_{(a, b)}}$. This completes the proof.

16. Now let us study the pseudogroups $\Gamma_{I_{1}^{a, b}}$ and $\Gamma_{I_{2}^{a, b b}}$ Let $\phi$ be any local transformation on $R^{2}$. Then for $z_{2}=p_{1}^{2} / p_{1}^{1}$ we have

$$
\begin{aligned}
& \phi^{(1)} *_{\gamma_{2}}=\left(\phi^{(1)} * p_{1}^{2}\right) /\left(\phi^{(1)} * p_{1}^{1}\right) \\
& =\left(\partial \phi_{2}(u) / \partial x_{1}\right) /\left(\partial \phi_{1}(u) / \partial x_{1}\right) \\
& =\left(\phi_{1}^{2} p_{1}^{1}+\phi_{2}^{2} p_{1}^{2}\right) /\left(\phi_{1}^{1} p_{1}^{1}+\phi_{2}^{1} p_{1}^{2}\right)
\end{aligned}
$$

where $\phi=\left(\phi_{1}, \phi_{2}\right)$ and $\phi_{j}^{i}=\partial \phi_{i} / \partial u_{j}$. Similarly we have

$$
\phi^{(1) *_{\partial 3}}=\left(\phi_{1}^{1} p_{2}^{1}+\phi_{2}^{1} p_{2}^{2}\right) /\left(\phi_{1}^{1} p_{1}^{1}+\phi_{2}^{1} p_{1}^{2}\right)
$$

and

$$
\phi^{(1)} *_{\partial_{4}}=\left(\phi_{1}^{2} p_{2}^{1}+\phi_{2}^{2} p_{2}^{2}\right) /\left(\phi_{1}^{1} p_{1}^{1}+\phi_{2}^{1} p_{1}^{2}\right)
$$

where $\jmath_{3}=p_{2}^{1} / p_{1}^{1}$ and $\jmath_{4}=p_{2}^{2} / p_{1}^{1}$.

PROPOSITION 16.1. $\quad \mathfrak{L}_{\Gamma_{I_{1}^{a}, b}}=\mathfrak{L}_{\Gamma_{I_{2}}^{a}, b}=\mathfrak{L}_{\mathfrak{P}_{(a, b)}}$ if $b \neq 0$. 
Proof. The differential invariant $I_{1}^{a, b}$ of $\Re_{(a, b)}$ is defined by

$$
\left.I_{1}^{a, b}=\left(\hat{\jmath}_{4}-\hat{\jmath}_{2} \partial_{3}\right) /\left(\partial_{2}\right)^{2}+a_{\partial_{2}}-b\right) .
$$

By (16.1), (16.2) and (16.3) we have

$$
\phi^{(1) *\left(z_{4}-z_{2} \partial_{3}\right)}=\left(\phi_{1}^{1} \phi_{2}^{2}-\phi_{2}^{1} \phi_{1}^{2}\right)\left(p_{1}^{1} p_{2}^{2}-p_{2}^{1} p_{1}^{2}\right) /\left(\phi_{1}^{1} p_{1}^{1}+\phi_{2}^{1} p_{1}^{2}\right)^{2}
$$

and

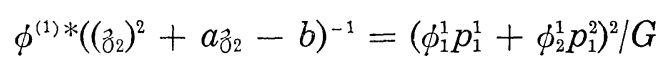

where

$$
G=\left(\phi_{1}^{2} p_{1}^{1}+\phi_{2}^{2} p_{1}^{2}\right)^{2}+a\left(\phi_{1}^{2} p_{1}^{1}+\phi_{2}^{2} p_{1}^{2}\right)\left(\phi_{1}^{1} p_{1}^{1}+\phi_{2}^{1} p_{1}^{2}\right)-b\left(\phi_{1}^{1} p_{1}^{1}+\phi_{2}^{1} p_{1}^{2}\right)^{2} .
$$

Then we get

$$
\phi^{(1) *} I_{1}^{a, b}=\left(\phi_{1}^{1} \phi_{2}^{2}-\phi_{2}^{1} \phi_{1}^{2}\right)\left(p_{1}^{1} p_{2}^{2}-p_{2}^{1} p_{1}^{2}\right) / G .
$$

On the other hand we have

$$
I_{1}^{a, b}=\left(p_{1}^{1} p_{2}^{2}-p_{2}^{1} p_{1}^{2}\right) /\left(\left(p_{1}^{2}\right)^{2}+a p_{1}^{2} p_{1}^{1}-b\left(p_{1}^{1}\right)^{2}\right) .
$$

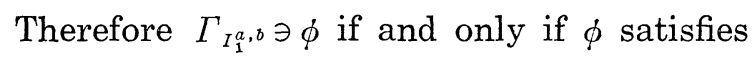

(16.4) $\quad\left(\phi_{1}^{1} \phi_{2}^{2}-\phi_{2}^{1} \phi_{1}^{2}\right)\left(p_{1}^{1} p_{2}^{2}-p_{2}^{1} p_{1}^{2}\right) / G=\left(p_{1}^{1} p_{2}^{2}-p_{2}^{1} p_{1}^{2}\right) /\left(\left(p_{1}^{2}\right)^{2}+a p_{1}^{2} p_{1}^{1}-b\left(p_{1}^{1}\right)^{2}\right)$. On $\tilde{J}^{1}\left(R^{2}, R^{2}\right)$, we have $p_{1}^{1} p_{2}^{2}-p_{2}^{1} p_{1}^{2} \neq 0$. Therefore the relation (16.4) is written by

$$
\left(\phi_{1}^{1} \phi_{2}^{2}-\phi_{2}^{1} \phi_{1}^{2}\right)\left(\left(p_{1}^{2}\right)^{2}+a p_{1}^{1} p_{1}^{2}-b\left(p_{1}^{1}\right)^{2}\right)=G .
$$

This relation identically holds as a polynomial with respect to the variables $p_{1}^{1}, p_{1}^{2}, p_{2}^{1}$ and $p_{2}^{2}$. Thus we can induce from (16.5) the following three relations among $\phi_{1}^{1}, \phi_{1}^{2}, \phi_{2}^{1}$ and $\phi_{2}^{2}$ :

$$
\begin{aligned}
& \phi_{1}^{1} \phi_{2}^{2}-\phi_{2}^{1} \phi_{1}^{2}=\left(\phi_{2}^{2}\right)^{2}+a \phi_{2}^{2} \phi_{2}^{1}-b\left(\phi_{2}^{1}\right)^{2}, \\
& b\left(\phi_{2}^{1} \phi_{1}^{2}-\phi_{1}^{1} \phi_{2}^{2}\right)=\left(\phi_{1}^{2}\right)^{2}+a \phi_{1}^{2} \phi_{1}^{1}-b\left(\phi_{1}^{1}\right)^{2}, \\
& a\left(\phi_{1}^{1} \phi_{2}^{2}-\phi_{2}^{1} \phi_{1}^{2}\right)=2 \phi_{1}^{2} \phi_{2}^{2}+a\left(\phi_{1}^{1} \phi_{2}^{2}+\phi_{2}^{1} \phi_{1}^{2}\right)-2 b \phi_{1}^{1} \phi_{2}^{1} .
\end{aligned}
$$

These relations $(16.6) \sim(16.8)$ are the defining equations of $\Gamma_{I_{1}^{a, b .}}$.

Let us now induce from these relations the defining equations of $\mathfrak{\Omega}_{\Gamma_{I_{1}^{a}, b}}$

Let $X(u)\left(\partial / \partial u_{1}\right)+Y(u)\left(\partial / \partial u_{2}\right)$ be any local cross-section of $\mathfrak{I}_{\Gamma_{I_{1}^{a}, b}}$ If $\phi_{t}$ is the local 1-parameter group of local transformations generated by $X(u)\left(\partial / \partial u_{1}\right)+Y(u)\left(\partial / \partial u_{2}\right), \phi_{t}$ satisfies $(16.6) \sim(16.8)$ and 


$$
\begin{aligned}
& d \phi_{t}(u) /\left.d t\right|_{t=0}=X(u)\left(\partial / \partial u_{1}\right)+Y(u)\left(\partial / \partial u_{2}\right) \\
& d \partial\left(\phi_{t}(u)\right)_{1} /\left.\partial u_{j} d t\right|_{t=0}=X_{u_{j}}(u) \\
& d \partial\left(\phi_{t}(u)\right)_{2} /\left.\partial u_{k} d t\right|_{t=0}=Y_{u_{k}}(u)
\end{aligned}
$$

where

$$
\phi_{t}(u)=\left(\left(\phi_{t}(u)\right)_{1},\left(\phi_{t}(u)\right)_{2}\right) .
$$

By these relations and by (16.6), (16.7), (16.8), we get

$$
\begin{aligned}
& X_{u_{1}}+Y_{u_{2}}=2 Y_{u_{2}}+a X_{u_{2}}, \\
& -b\left(X_{u_{1}}+Y_{u_{2}}\right)=a Y_{u_{1}}-2 b X_{u_{1}}, \\
& a\left(X_{u_{1}}+Y_{u_{2}}\right)=2 Y_{u_{1}}+a\left(X_{u_{1}}+Y_{u_{2}}\right)-2 b X_{u_{2}} .
\end{aligned}
$$

From (16.9) and (16.11), we obtain

$$
\begin{aligned}
& X_{u_{1}}=a X_{u_{2}}+Y_{u_{2}}, \\
& Y_{u_{1}}=b X_{u_{2}} .
\end{aligned}
$$

Since (16.10) is induced from (16.11), the relations (16.12) and (16.13) are equivalent to the relations (16.9), (16.10) and (16.11). This proves that (16.12) and (16.13) are the defining equations of $\mathfrak{L}_{\Gamma_{I_{1}^{a}, b}}$ and this means that $\mathfrak{\Omega}_{\Gamma_{I_{1}^{a}, b}}=\mathfrak{\Omega}_{\mathfrak{P}_{(a, b)}}$.

Next let us consider the defining equations of $\mathfrak{I}_{\Gamma_{I_{2}^{a}, b}}$. We have

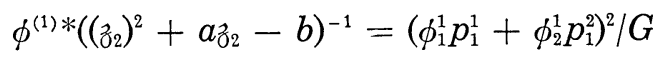

and

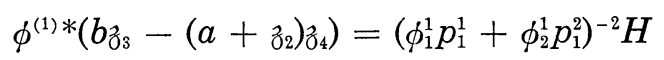

where

$$
\begin{aligned}
H= & b\left(\phi_{1}^{1} p_{2}^{1}+\phi_{2}^{1} p_{2}^{2}\right)\left(\phi_{1}^{1} p_{1}^{1}+\phi_{2}^{1} p_{1}^{2}\right)-a\left(\phi_{1}^{1} p_{1}^{1}+\phi_{2}^{1} p_{1}^{2}\right)\left(\phi_{1}^{2} p_{2}^{1}+\phi_{2}^{2} p_{2}^{2}\right) \\
& -\left(\phi_{1}^{2} p_{1}^{1}+\phi_{2}^{2} p_{1}^{2}\right)\left(\phi_{1}^{2} p_{2}^{1}+\phi_{2}^{2} p_{2}^{2}\right) \\
= & \left(b\left(\phi_{1}^{1}\right)^{2}-a \phi_{1}^{1} \phi_{1}^{2}-\left(\phi_{1}^{2}\right)^{2}\right) p_{1}^{1} p_{2}^{1}+\left(b \phi_{1}^{1} \phi_{2}^{1}-a \phi_{2}^{1} \phi_{1}^{2}-\phi_{2}^{2} \phi_{1}^{2}\right) p_{2}^{1} p_{1}^{2} \\
& +\left(b \phi_{2}^{1} \phi_{1}^{1}-a \phi_{1}^{1} \phi_{2}^{2}-\phi_{1}^{2} \phi_{2}^{2}\right) p_{1}^{1} p_{2}^{2}+\left(b\left(\phi_{2}^{1}\right)^{2}-a \phi_{2}^{1} \phi_{2}^{2}-\left(\phi_{2}^{2}\right)^{2}\right) p_{1}^{2} p_{2}^{2} .
\end{aligned}
$$

Since $I_{2}^{a, b}=\left(\left(a+z_{2}\right) \vec{z}_{4}-b z_{3}\right) /\left(\left(z_{2}\right)^{2}+a_{z_{2}}-b\right)$, by easy calculations we see that $\phi$ satisfies $\phi^{(1) *} I_{2}^{a, b}=I_{2}^{a, b}$ if and only if

$$
\left(\left(p_{1}^{2}\right)^{2}+a p_{1}^{1} p_{1}^{2}-b\left(p_{1}^{1}\right)^{2}\right) H=\left(b p_{1}^{1} p_{2}^{1}-a p_{1}^{1} p_{2}^{2}-p_{1}^{2} p_{2}^{2}\right) G .
$$

This relation holds identically as a polynomial with respect to the variables $p_{1}^{1}, p_{1}^{2}, p_{2}^{1}$ and $p_{2}^{2}$. Therefore we get the following relations (16.15) (16.22) among $\phi_{1}^{1}, \phi_{1}^{2}, \phi_{2}^{1}$ and $\phi_{2}^{2}$ : 


$$
\begin{aligned}
& b\left(\phi_{1}^{1}\right)^{2}-a \phi_{1}^{1} \phi_{1}^{2}-\left(\phi_{1}^{2}\right)^{2}+a\left(b \phi_{1}^{1} \phi_{2}^{1}-a \phi_{2}^{1} \phi_{1}^{2}-\phi_{2}^{2} \phi_{1}^{2}\right) \\
& \quad=b\left(\left(\phi_{2}^{2}\right)^{2}+a \phi_{2}^{2} \phi_{2}^{1}-b\left(\phi_{2}^{1}\right)^{2}\right), \\
& a\left(b\left(\phi_{1}^{1}\right)^{2}-a \phi_{1}^{1} \phi_{1}^{2}-\left(\phi_{1}^{2}\right)^{2}\right)-b\left(b \phi_{1}^{1} \phi_{2}^{1}-a \phi_{2}^{1} \phi_{1}^{2}-\phi_{2}^{2} \phi_{1}^{2}\right) \\
& \quad=b\left(2 \phi_{1}^{2} \phi_{2}^{2}+a\left(\phi_{1}^{1} \phi_{2}^{2}+\phi_{2}^{1} \phi_{1}^{2}\right)-2 b \phi_{1}^{1} \phi_{2}^{1}\right) \\
& -b\left(b\left(\phi_{1}^{1}\right)^{2}-a \phi_{1}^{1} \phi_{1}^{2}-\left(\phi_{1}^{2}\right)^{2}\right)=b\left(\left(\phi_{1}^{2}\right)^{2}+a \phi_{1}^{2} \phi_{1}^{1}-b\left(\phi_{1}^{1}\right)^{2}\right) \\
& b \phi_{1}^{1} \phi_{2}^{1}-a \phi_{2}^{1} \phi_{1}^{2}-\phi_{2}^{2} \phi_{1}^{2}=0 \\
& b \phi_{2}^{1} \phi_{1}^{1}-a \phi_{1}^{1} \phi_{2}^{2}-\phi_{1}^{2} \phi_{2}^{2}+a\left(b\left(\phi_{2}^{1}\right)^{2}-a \phi_{2}^{1} \phi_{2}^{2}-\left(\phi_{2}^{2}\right)^{2}\right) \\
& \quad=-\left(2 \phi_{1}^{2} \phi_{2}^{2}+a\left(\phi_{1}^{1} \phi_{2}^{2}+\phi_{2}^{1} \phi_{1}^{2}\right)-2 b \phi_{1}^{1} \phi_{2}^{1}\right) \\
& \quad-a\left(\left(\phi_{2}^{2}\right)^{2}+a \phi_{2}^{2} \phi_{2}^{1}-b\left(\phi_{2}^{1}\right)^{2}\right), \\
& a\left(b \phi_{2}^{1} \phi_{1}^{1}-a \phi_{1}^{1} \phi_{2}^{2}-\phi_{1}^{2} \phi_{2}^{2}\right)-b\left(b\left(\phi_{2}^{1}\right)^{2}-a \phi_{2}^{1} \phi_{2}^{2}-\left(\phi_{2}^{2}\right)^{2}\right) \\
& \quad=-\left(\left(\phi_{1}^{2}\right)^{2}+a \phi_{1}^{2} \phi_{1}^{1}-b\left(\phi_{1}^{1}\right)^{2}\right) \\
& \quad-a\left(2 \phi_{1}^{2} \phi_{2}^{2}+a\left(\phi_{1}^{1} \phi_{2}^{2}+\phi_{2}^{1} \phi_{1}^{2}\right)-2 b \phi_{1}^{1} \phi_{2}^{1}\right), \\
& b\left(b \phi_{2}^{1} \phi_{1}^{1}-a \phi_{1}^{1} \phi_{2}^{2}-\phi_{1}^{2} \phi_{2}^{2}\right)=a\left(\left(\phi_{1}^{2}\right)^{2}+a \phi_{1}^{2} \phi_{1}^{1}-b\left(\phi_{1}^{1}\right)^{2}\right) \\
& b\left(\phi_{2}^{1}\right)^{2}-a \phi_{2}^{1} \phi_{2}^{2}-\left(\phi_{2}^{2}\right)^{2}=-\left(\left(\phi_{2}^{2}\right)^{2}+a \phi_{2}^{2} \phi_{2}^{1}-b\left(\phi_{2}^{1}\right)^{2}\right) .
\end{aligned}
$$

These relations are the defining equations of $\Gamma_{I_{2}^{a}, b}$

Let us induce the defining equations of $\mathfrak{L}_{\Gamma_{I_{2}}^{a, b}}$ from the above relations $(16.15) \sim(16.22)$. Let $X(u)\left(\partial / \partial u_{1}\right)+Y(u)\left(\partial / \partial u_{2}\right)$ be any local crosssection of $\mathfrak{L}_{\Gamma_{I_{2}^{a}, b}}$. Then from (16.19) we get

$$
Y_{u_{1}}=b X_{u_{2}} \text {. }
$$

From (16.16) we get $b X_{u_{1}}=a b X_{u_{2}}+b Y_{u_{2}}$ and so

$$
X_{u_{1}}=a X_{u_{2}}+Y_{u_{2}}
$$

because $b \neq 0$. It is easy to check that $X(u)$ and $Y(u)$ satisfy the other relations induced from $(16.15) \sim(16.22)$ if they satisfy the relations (16.23) and (16.24). Therefore $X(u)\left(\partial / \partial u_{1}\right)+Y(u)\left(\partial / \partial u_{2}\right)$ is a local cross-section of $\mathfrak{\Omega}_{\Gamma_{I_{2}^{a}}^{a}, b}$ if and only if it satisfies (16.23) and (16.24). This completes the proof of Proposition 16.1.

TheORem 16.2. Let $F\left(z_{1}, z_{2}\right)$ be a non-constant real rational expression of the variables $z_{1}$ and $z_{2}$. We set $\lambda(x)=F\left(I_{1}^{a, b}\left(j_{x}^{1}(f)\right), I_{2}^{a, b}\left(j_{x}^{1}(f)\right)\right)$ and consider the single differential equation $P(F): F\left(I_{1}^{a, b}, I_{2}^{a, b}\right)=\lambda$ around the point $p=j_{x_{0}}^{1}(f)$. Then the automorphism pseudogroup $\mathfrak{U}(P(F))$ of $P(F)$ coincides with the pseudogroup $\mathfrak{P}_{(a, b)}$ around $\beta^{1}(p)$ and near the identity if $b \neq 0$. 
Proof. Since $I_{1}^{a, b}$ and $I_{2}^{a, b}$ are functionally independent, by Proposition 16.1 and Corollary 15.3, we have $\mathfrak{L}_{\Gamma(F)}=\mathfrak{L}_{\mathfrak{F}_{(a, b)}}$ where $\Gamma(F)=\{\phi \in$ $\left.\mathscr{A}\left(R^{2}\right) ; \phi^{(1) *} F\left(I_{1}^{a, b}, I_{2}^{a, b}\right)=F\left(I_{1}^{a, b}, I_{2}^{a, b}\right)\right\}$. Then by Lemma 14.1 , we get $\mathfrak{R}_{\mathfrak{r}(P(F))}=$ $\mathfrak{R}_{\mathfrak{p}_{(a, b)}}$. This completes the proof of Theorem 16.2.

\section{§6. Foliations and differential equations}

17. Let us consider a foliation $\mathfrak{\mho}$ on a manifold $M$ and denote by $T(\mathfrak{F})$ the tangent bundle to $\mathfrak{F}$.

Definition 17.1. For a foliation $\mathfrak{F}$, if there exist 1 -forms $\omega_{1}, \cdots, \omega_{q}$ on $M$ satisfying the following conditions (1), (2) and (3), then the pair $(\mathfrak{F}, \omega)$ is called a generalized Lie foliation where $\omega=\left(\omega_{1}, \cdots, \omega_{q}\right)$ :

(1) $q=\operatorname{codim} \mathfrak{\mho}$.

(2) $T(\widetilde{\mho}) \ni X$ if and only if $\omega(X)=0$.

(3) $d \omega_{i}=\sum_{j<k} c_{i j k} \omega_{j} \wedge \omega_{k}$ where all $c_{i j k}$ are functions on $M$. If all $c_{i j k}$ are constants, $(\mathfrak{\mho}, \omega)$ is called a Lie foliation.

Now consider a differential operator $P(x, D)$ of infinite type at any $x$ with the structure vector $(a, b)$. Then we have a system of differential equations $P: P(x, D) u=0$ which is written on $\widetilde{J}^{1}\left(R^{2}, R^{2}\right)$ by (9.1). By the pseudoellipticity of (9.1), it is also written by the following normal form

$$
\begin{aligned}
& p_{1}^{1}=H^{1}\left(x_{1}, x_{2}, p_{2}^{1}, p_{2}^{2}\right), \\
& p_{1}^{2}=H^{2}\left(x_{1}, x_{2}, p_{2}^{1}, p_{2}^{2}\right) .
\end{aligned}
$$

Lemma 17.1. The set of integral points $I(P)$ of (9.1) or (17.1) is a 6dimensional regular submanifold of $J^{1}\left(R^{2}, R^{2}\right)$.

Proof. The vector fields $Z_{1}=\left(a p_{1}^{1}+p_{1}^{2}\right)\left(\partial / \partial p_{1}^{1}\right)+b p_{1}^{1}\left(\partial / \partial p_{1}^{2}\right)+\left(a p_{2}^{1}+p_{2}^{2}\right)$ $\cdot\left(\partial / \partial p_{2}^{1}\right)+b p_{2}^{1}\left(\partial / \partial p_{2}^{2}\right)$ and $Z_{2}=p_{1}^{1}\left(\partial / \partial p_{1}^{1}\right)+p_{1}^{2}\left(\partial / \partial p_{1}^{2}\right)+p_{2}^{1}\left(\partial / \partial p_{2}^{1}\right)+p_{2}^{2}\left(\partial / \partial p_{2}^{2}\right)$ on $\tilde{J}^{1}\left(R^{2}, R^{2}\right)$ are linearly independent at each point because $p_{1}^{1} p_{2}^{2}-p_{2}^{1} p_{1}^{2} \neq 0$. We set $D_{p}^{(1)}=\left(\mathcal{Q}_{\mathfrak{U}(P)}^{(1)}\right)_{p} /\left(\mathcal{Q}_{\mathfrak{Q}(P)}^{(1)}\right)_{p}^{0}$ and consider the correspondence $D^{(1)}$ : $\tilde{J}^{1}\left(R^{2}, R^{2}\right) \ni p \rightarrow D_{p}^{(1)} \subset T_{p}\left(\tilde{J}^{1}\left(R^{2}, R^{2}\right)\right)$. Then by Proposition $6.2, D^{(1)}$ is a 4 dimensional involutive distribution on $\tilde{J}^{1}\left(R^{2}, R^{2}\right)$ generated by $Z_{1}, Z_{2}, Z_{3}=$ $\partial / \partial u_{1}$ and $Z_{4}=\partial / \partial u_{2}$. Since the image of the map $j^{1}(s): U \rightarrow \tilde{J}^{1}\left(R^{2}, R^{2}\right)$ for any solution $s: U \rightarrow R^{2}$ of $P$ is transversal to $D^{(1)}$ and $\operatorname{dim}\left(\operatorname{Im}\left(j^{1}(s)\right)\right)=2$, we see that $I(P)$ is a 6-dimensional regular submanifold of $\tilde{J}^{1}\left(R^{2}, R^{2}\right)$ defined by $p_{1}^{1}=H^{1}\left(x_{1}, x_{2}, p_{2}^{1}, p_{2}^{2}\right)$ and $p_{1}^{2}=H^{2}\left(x_{1}, x_{2}, p_{2}^{1}, p_{2}^{2}\right)$. Since $\tilde{J}^{1}\left(R^{2}, R^{2}\right)$ is open in $J^{1}\left(R^{2}, R^{2}\right)$, the proof of Lemma 17.1 is completed. 
Let us consider the distribution $\tilde{E}$ on $\tilde{J}^{1}\left(R^{2}, R^{2}\right)$ generated by the following vector fields $E_{1}$ and $E_{2}$ :

$$
\begin{aligned}
& E_{1}=\partial / \partial x_{1}+p_{1}^{1}\left(\partial / \partial u_{1}\right)+p_{1}^{2}\left(\partial / \partial u_{2}\right), \\
& E_{2}=\partial / \partial x_{2}+p_{2}^{1}\left(\partial / \partial u_{1}\right)+p_{2}^{2}\left(\partial / \partial u_{2}\right) .
\end{aligned}
$$

Since $\left[E_{1}, E_{2}\right]=0, \tilde{E}$ is involutive. For any local map $f$ of $R^{2}$ to $R^{2}$, the vector fields $E_{1}$ and $E_{2}$ are tangent to the image of the map $j^{1}(f)$ at each point of it. Since $P$ is pseudoinvolutive, we have $I(P)=S=\left\{j_{x}^{1}(s)\right.$; $s \in \subseteq(P), x \in$ the domain of $s\}$. By Lemma $17.1, I(P)$ is a regular submanifold of $\tilde{J}^{1}\left(R^{2}, R^{2}\right)$. Thus we can obtain the involutive distribution $E$ on $I(P)$ by restricting $\tilde{E}$ to $I(P)$.

Proposition 17.2. The foliation $\mathfrak{F}$ on $S=I(P)$ given by $E$ is a generalized Lie foliation of codim 4.

Proof. The vector fields $E_{1}, E_{2}, Z_{1}, Z_{2}, Z_{3}, Z_{4}$ on $\tilde{J}^{1}\left(R^{2}, R^{2}\right)$ are tangent to $S$ and linearly independent at each point of $S$. By Lemma 17.1 these vector fields define a complete parallelism on $S$.

Let $L$ be any leaf of $\widetilde{F}$. Then for any point $p \in L$, there exists a neighbourhood $\mathfrak{U}$ of $p$ such that $L \cap \mathfrak{U}$ is the image of the map $j^{1}(s)$ for a solution $s$ of $P$. Conversely for a solution $s$ of $P$, the image of $j^{1}(s)$ is an open subset of a leaf of $\widetilde{F}$. By Proposition 6.2, $Z_{1}, Z_{2}, Z_{3}$ and $Z_{4}$ are cross-sections of $\mathfrak{Q}_{\mathfrak{q}(P)} . \quad E_{1}$ and $E_{2}$ are tangent to any leaf $L$ of $\widetilde{F}$ at any point. Thus we see that $\left[E_{i}, Z_{j}\right]$ is also tangent to any leaf of $\widetilde{F}$, that is, at any point of $S,\left[E_{i}, Z_{j}\right]$ is a linear combination of $E_{1}$ and $E_{2}$. On the other hand, $\left[Z_{i}, Z_{j}\right]$ is clearly a cross-section of $\mathfrak{Q}_{\mathfrak{x}(P)}$, that is, $\left[Z_{i}, Z_{j}\right]$ is a linear combination of $Z_{1}, Z_{2}, Z_{3}$ and $Z_{4}$ at any point of $S$.

Let $\omega_{1}, \omega_{2}, \omega_{3}, \omega_{4}$, be 1 -forms on $S$ defined by $\omega_{i}\left(Z_{j}\right)=\delta_{i j}$ and $\omega_{i} \mid E=0$. Then it is easy to check that

$$
d \omega_{i}=\sum_{j k} c_{i j k} \omega_{j} \wedge \omega_{k}
$$

and

$$
T(\widetilde{\mho}) \ni X \quad \text { if and only if } \omega(X)=0
$$

where all $c_{i \jmath k}$ are functions on $S$ and $\omega=\left(\omega_{1}, \omega_{2}, \omega_{3}, \omega_{4}\right)$. This shows that the pair $(\mathfrak{F}, \omega)$ is a generalized Lie foliation of codim 4. This completes the proof of Proposition 17.2.

18. Let $f$ be a submersion of $R^{2}$ to $R^{2}$ and let us consider a single differential equation 


$$
P(F): \quad F\left(I_{1}^{a, b}, I_{2}^{a, b}\right)=\lambda
$$

on $\tilde{J}^{1}\left(R^{2}, R^{2}\right)$ where $F\left(z_{1}, z_{2}\right)$ is a non-constant real rational expression of the variables $z_{1}$ and $z_{2}$ and $\lambda(x)=F\left(I_{1}^{a, b}\left(j_{x}^{1}(f)\right), I_{2}^{a, b}\left(j_{x}^{1}(f)\right)\right)$. The automorphism pseudogroup $\mathfrak{A}(P(F))$ is, by Theorem $16.2, \mathfrak{P}_{(a, b)}$ near the identity if $b \neq 0$.

Assume that $b \neq 0, I(P(F))$ is a regular submanifold and $P(F)$ is pseudoinvolutive i.e. $I(P(F))=S(P(F))$. Let $s: U \rightarrow R^{2} \in S(P(F))$ and denote by $G^{1}(s)$ the image of the map $j^{1}(s): U \rightarrow \tilde{J}^{1}\left(R^{2}, R^{2}\right)$. If we denote by $\widetilde{\Im}_{p}$ the maximal integral manifold of $D^{(1)}$ through $p \in \tilde{J}^{1}\left(R^{2}, R^{2}\right)$, then $D^{(1)}(s)=\cup_{n \in G^{1}(s)} \widetilde{J}_{p}$ is a regular submanifold of $S(P(F))$. Denote by $S^{0}(P(F))$ the connected component of $S(P(F))$ containing $G^{1}(f)$.

Proposition 18.1. There exists a foliation $\mathfrak{F}$ on $S^{\circ}(P(F))$ satisfying the following conditions:

(i) $\operatorname{codim} \tilde{F}=1$.

(ii) On each leaf $L$ of $\widetilde{F}$, there exists a generalized Lie foliation $\left(\widetilde{\mho}_{L}, \omega_{L}\right.$, of codim 4 .

Proof. Let $L$ be a maximal connected subset of $S^{0}(P(F)$ ) which is locally given by $D^{(1)}(s)$ for some $s \in \mathcal{S}(P(F))$. Denote by $\widetilde{F}$ the family of all such subsets of $S^{0}(P(F))$. Then it is clear that $S^{0}(P(F))=\cup_{L \in \mathbb{F}} L$.

If $L_{1}$ and $L_{2}$ are elements of $\widetilde{\mho}$ with $L_{1} \cap L_{2} \neq \phi$, then for $p \in L_{1} \cap L_{2}$ there exists an element $s \in \mathbb{S}(P(F))$ such that $j_{x}^{1}(s)=p$. Since $D^{(1)}(s) \subset L_{1}$. $D^{(1)}(s) \subset L_{2}$ and $\operatorname{dim} D^{(1)}(s)=\operatorname{dim} L_{1}=\operatorname{dim} L_{2}$, it is clear that $L_{1}=L_{2}$. Therefore $\mathfrak{\mho}$ is a foliation on $S^{0}(P(F))$ and codim $\mathfrak{F}=1$ because $\operatorname{dim} S^{0}(P(F))=7$ and $\operatorname{dim} L=6$ for $L \in \widetilde{\mho}$.

Since the vector fields $E_{1}=\partial / \partial x_{1}+p_{1}^{1}\left(\partial / \partial u_{1}\right)+p_{1}^{2}\left(\partial / \partial u_{2}\right)$ and $E_{2}=\partial / \partial x_{2}$ $+p_{2}^{1}\left(\partial / \partial u_{1}\right)+p_{2}^{2}\left(\partial / \partial u_{2}\right)$ are tangent to $G^{1}(s)$, they define a 2-dimensional involutive distribution $D_{L}$ on $L$ i.e. the foliation $\mathfrak{F}_{L}$ on $L$. Furthermore the vector fields $Z_{1}, Z_{2}, Z_{3}$ and $Z_{4}$ are tangent to $D^{(1)}(s)$ at each point of $D^{(1)}(s)$ for $s \in \mathfrak{S}(P(F))$ because $\mathfrak{R}_{\mathfrak{r}(P(F))}=\mathfrak{R}_{\mathfrak{P}_{(a, b)}}$. Therefore, in particular, they are tangent to $L$ at each point of $L$. Define the vector valued 1form $\omega_{L}=\left(\omega_{L}^{1}, \omega_{L}^{2}, \omega_{L}^{3}, \omega_{L}^{4}\right)$ by

$$
\begin{aligned}
& \omega_{L}^{i} \mid D_{L}=0, \\
& \omega_{L}^{i}\left(Z_{j}\right)=\delta_{i j} .
\end{aligned}
$$

Then we see that the pair $\left(\widetilde{\mho}_{L}, \omega_{L}\right)$ is a generalized Lie foliation on $L$ of codim 4. This completes the proof of Proposition 18.1. 


\section{REFERENCES}

[1] C. Chevalley, Theory of Lie groups I, Princeton University Press, 1946.

[2] I. M. Singer and S. Sternberg, The infinite groups of Lie and Cartan, J. Analyse Math., 15 (1965), 1-114.

[ 3 ] K. Ueno, Existence and equivalence theorems of automorphic systems, Publ. RIMS, Kyoto Univ., 11 (1976), 461-482.

[4] K. Ueno, Projective structures of elliptic differential operators, Nagoya Math. J., 99 (1985), 111-130.

Tokyo University of Fisheries

Minato-ku, Tokyo 108, Japan 